Canadian University Music Review

Revue de musique des universités canadiennes

\title{
Présence wagnérienne dans le film Invincible (2001) de Werner Herzog
}

\section{Audrée Descheneaux}

Volume 24, numéro 1, 2003

URI : https://id.erudit.org/iderudit/1014670ar

DOI : https://doi.org/10.7202/1014670ar

Aller au sommaire du numéro

\section{Éditeur(s)}

Canadian University Music Society / Société de musique des universités canadiennes

ISSN

0710-0353 (imprimé)

2291-2436 (numérique)

Découvrir la revue

\section{Citer cet article}

Descheneaux, A. (2003). Présence wagnérienne dans le film Invincible (2001) de Werner Herzog. Canadian University Music Review / Revue de musique des universités canadiennes, 24(1), 30-61. https://doi.org/10.7202/1014670ar

\section{Résumé de l'article}

Dans son film Invincible (2001), le réalisateur allemand Werner Herzog met en scène la légende de Zishe Breibart, un Juif doté d'une force surhumaine. La théâtralité des images filmées par Herzog et la musique originale du film composée par Hans Zimmer concourent, selon l'auteure, à accentuer la dimension héroïque du personnage et à évoquer l'opéra wagnérien. Après avoir mis en parallèle les procédés de composition de la musique du film et de l'écriture postromantique, l'article propose une analyse comparative entre la musique et les images pour démontrer comment la musique du film sert de support privilégié au mythe wagnérien. L'article s'attarde enfin aux répercussions du subterfuge mis en scène par Herzog, qui consiste à faire d'un Juif un héros national à l'aube du nazisme.
All Rights Reserved @ Canadian University Music Society / Société de musique des universités canadiennes, 2005
Ce document est protégé par la loi sur le droit d'auteur. L'utilisation des services d'Érudit (y compris la reproduction) est assujettie à sa politique d'utilisation que vous pouvez consulter en ligne.

https://apropos.erudit.org/fr/usagers/politique-dutilisation/ 


\title{
PRÉSENCE WAGNÉRIENNE DANS LE FILM INVINCIBLE (2001) DE WERNER HERZOG
}

\author{
Audrée Descheneaux
}

Dès ses débuts, le cinéma de fiction a toujours mis en scène des héros aux nobles intentions opposés à des protagonistes aux vils desseins. Contrairement au cinéma américain où l'on illustrait avec souci de réalisme les conflits entre colonisateurs blancs et Indiens, le cinéma allemand s'intéressait davantage aux récits fantastiques sur fond d'action psychologique. Le premier film participant de cette esthétique, Der Student von Prag (1913) de Paul Wegener, se présente comme une variation de Faust, dans lequel le héros vend son reflet au diable ${ }^{1}$. Le climat qui s'en dégage est empreint d'angoisse et de clair-obscur très près du romantisme. Dans un style tout aussi expressionniste, Der Golem (1916) de Wegener raconte l'histoire d'un héros juif doté d'une force surhumaine et dont la mission est de délivrer son peuple ${ }^{2}$. Aujourd'hui encore, peu de choses ont changé dans les cinémas américain et allemand quant à la représentation du héros. Le film Invincible (2001, version originale anglaise) de Werner Herzog en est un exemple manifeste. De même, les parallèles entre le héros romantique d'Invincible et celui de Der Golem impressionnent par leur inspiration issue d'une même source mythologique. Der Student von Prag et Der Golem, qui contiennent déjà en eux la dualité entre le pur romantisme allemand et la question du sauveur des Juifs, semblent se condenser dans Invincible. En effet, nous verrons que le héros principal, un Juif, cumule également toutes les qualités du grand héros germanique. Ainsi, il résout à la fois l'attente messianique d'un peuple et l'idéal allemand. Le tout est campé dans un tableau historique préparant l'avènement du Führer et acclamant la gloire future de la race aryenne.

Pareillement à ces cinéastes, Wagner apparaît dans l'opéra allemand comme la figure la plus représentative de l'utilisation du mythe comme procédé

1 Je réfère ici à la première version de Der Student von Prag de Paul Wegener. Il en existe une seconde réalisée par Henrik Galeen en 1926. Les deux films, inspirés de la même légende folklorique, racontent l'histoire d'un jeune étudiant qui conclut avec le diable (Scapinelli) un pacte lui permettant de trouver l'amour et la célébrité. En échange, Scapinelli emporte avec lui le reflet de l'étudiant dans un miroir et devient son double.

2 Il existe d'autres versions de Der Golem, dont celle de 1915 de Hendrik Galeen et celles de 1917 et de 1920 de Wegener. Il s'agit, comme pour Der Student von Prag, de films muets de facture expressionniste. Der Golem est inspiré d'une légende allemande. Au XVI à une statue d'argile dont la mission sera de défendre les Juifs persécutés. Ce thème du monstre à la force surhumaine semble avoir été un sujet privilégié pour le premier cinéma allemand. Il incarne parfaitement le mythe du héros terrifiant évoluant dans une atmosphère fantastique et insolite (Bellan 2001, 21-22). 
structurant. Le réservoir de mythes au cœur de l'imaginaire des créateurs allemands se lirait dans leur cinéma à la manière d'un métarécit qui raconterait les origines et la destinée de ce peuple. L'idée de recréer la dimension de l'opéra à l'écran fut au cœur des premières recherches sur la diffusion et la démocratisation du cinéma auprès du public. N'ayant pu se réaliser faute d'avancées techniques, cette idée est restée en suspens, sans du moins être totalement abandonnée ${ }^{3}$. L'utilisation des musiques d'opéra, l'emploi de certains clichés dramatiques ou la présence d'une scène d'opéra témoignent autant de cette persistance de l'opéra au sein du film. Et si le film de Herzog, sur une musique originale de Hans Zimmer et de Klauss Badelt, atteste l'influence de l'opéra, il contient en lui-même certains clichés opératiques de la représentation du héros. À ce sujet, Herzog, qui a également mis en scène une vingtaine d'opéras, dont Lohengrin (1987) et Tannhäuser (1997-2000) de Wagner, ne se gêne pas pour revendiquer cette filiation. La présence wagnérienne apparaît déjà dans ses films antérieurs, dont Fitzcarraldo (1982) qui raconte le rêve d'un amant de la musique voulant construire un opéra dans la forêt péruvienne pour y faire chanter Caruso, ce qui n'est pas sans rappeler le projet du Festspielhaus et la mégalomanie de Wagner (Bellan 2001, 91).

Le présent article s'emploie à relever de tels emprunts à l'opéra et à l'imaginaire wagnériens : l'étude de l'action historique et mythique du film Invincible de Werner Herzog vise à démontrer la persistance d'un imaginaire de type wagnérien à travers la cinématographie allemande au lendemain de la Seconde Guerre, ce qui passe par l'analyse des relations entre les images et la musique. Les analyses de trois scènes d'Invincible s'orientent autour du principal point d'ancrage narratif du film, c'est-à-dire le héros lui-même, qui se présente comme le nouveau Siegfried. L'article évalue également les relations qu'entretient ce personnage avec la dramaturgie wagnérienne et la pertinence de l'utilisation du mythe par Werner Herzog.

\section{LA DIMENSION MYTHOLOGIQUE DU HÉROS}

\section{Argument du film}

Avant de présenter Invincible, je débuterai par quelques mots sur son réalisateur, Werner Herzog, considéré encore aujourd'hui par la critique de cinéma comme l'un des réalisateurs les plus visionnaires de sa génération. Né à Munich le 5 septembre 1942, Herzog grandit dans un village de Bavière isolé du monde urbain et expérimente tardivement la technologie moderne (premier appel téléphonique à l'âge de 17 ans). Fasciné par l'invention du cinéma, il réalise ses premiers films dès l'âge de 19 ans. Il reçoit plusieurs récompenses, dont le Grand prix spécial du jury et le Prix de la critique internationale de Cannes en 1975 pour Jeder fur sich und Gott Gegen Alle (Les nains aussi ont commencé

3Dans Opérascope. Le film-opéra en Amérique, Réal La Rochelle $(2003,111)$ émet l'hypothèse que le rêve de l'opéra filmé d'Edison n'a pu se réaliser faute d'avancées techniques dans la synchronisation de l'image et du son filmique. D'autre part, le développement du théâtre musical de Broadway aurait éclipsé le goût pour l'opéra à l'européenne. 
petits), ainsi que le prix de la meilleure mise en scène pour Fitzcarraldo (1982). Sa filmographie contient aujourd' hui plus de quarante films et documentaires.

Invincible, le quarante-quatrième film de Herzog, marque le retour du cinéaste à la réalisation de films de fiction après une période de dix ans où il se dédie exclusivement au documentaire. Tourné en langue anglaise, Invincible est présenté en 2001 au Venice Internationale Film Festival. Critiqué principalement pour ses lenteurs et ses longs plans fixes (Sullivan 2002) qui firent pourtant l'originalité des films de Herzog, à mi-chemin entre le réalisme contemplatif et le théâtre, Invincible ne plaît pas autant que les films précédents dont il conserve cependant certaines caractéristiques majeures : héros hors du commun, paysages rappelant une nature non civilisée, puissance visionnaire, action initiatique. Dans le Chicago Sun Times, Roger Ebert (2002) rend hommage à l'expérience cinématographique unique qu'offre le visionnage d'un film de Herzog, tandis que Gilles Lyon-Caen (2002), dans Objectif cinéma, qualifie Invincible de "film itinérant », en mouvance infinie.

Invincible relate le récit vécu de Zishe Breitbart, un homme doté d'une force surhumaine qui deviendra le prophète de son peuple. Les premières images du film montrent une place du marché dans un village polonais où réside une communauté juive. Les premières paroles prononcées sont celles d'un petit garçon juif d'une grande intelligence racontant la fable du coq à table : «Don't ever think, said the sage, that by eating like a man, like other men at the table, a rooster ceases to be what he is » $(2$. "A Rooster at the Table », 0.04.00)4. Cette fable est au cœur de la quête d'authenticité qui marque l'évolution des personnages. Le frère du petit garçon est un colosse nommé Zishe, qui s'étonne de la sagesse de son frère et qui possède un don exceptionnel, celui de la force physique ${ }^{5}$. À partir de cette admiration mutuelle prise comme modèle entre les deux frères, les relations entre les personnages au cours du film remettront en question cet équilibre entre l'esprit et le corps en créant une dualité.

Les premières manifestations de la force physique de Zishe ont lieu dès la fin du deuxième tableau («A Rooster at the Table ») pour démontrer la nature impulsive du héros attaqué sur sa condition par les Allemands. Dans le troisième tableau ("Hercules Has a Challenger »), Zishe doit payer une dette en affrontant au cirque un colosse masqué du nom d'Hercule, qu'il soulève et terrasse. De ces épisodes suivra une prise de conscience chez le héros de sa force surhumaine et du sentiment de devoir accomplir une mission dans le monde. Un intermédiaire agent d'artiste, côtoyant les cirques à la recherche de phénomènes, ouvre la possibilité à Zishe de se rendre à Berlin pour y travailler. Il lui montre des images de la ville filmées par le cinématographe. Le héros, impressionné par les avancées techniques du

4 « Surtout, ne pense jamais, lui dit le sage, qu'en mangeant comme un homme avec les autres hommes à table, un coq cesse d'être ce qu'il est. » Les traductions sont tirées de la version française du film, accessible sur DVD. Les références au DVD à la suite des citations donnent les numéros de tableaux (chapitres) et leur titre. Le minutage correspond au début de la citation dans le film.

5Pour incarner Zishe, Herzog choisit Youko Ahola, un athlète non acteur nommé deux fois champion du monde de poids lourd et considéré comme l'homme le plus fort du monde (Ebert 2002). 
monde civilisé, décide de partir avec l'aval du père, qui lui fait cependant cette recommandation riche de sens : "You will go out into the world, but don't ever forget who you are ${ }^{6}$ " (5. "Zishe's Gift of Strength », 0.21.00).

Ces scènes à la campagne permettent en un premier temps d'identifier les origines et le don exceptionnel de ce personnage. Zishe, dont le nom en allemand se rapproche des verbes zischen (siffler) et zischeln (marmonner), renvoie à deux manières de s'exprimer sans avoir recours à la clarté des mots. En effet, Zishe n'est pas un homme d'esprit qui s'exprime par la parole, mais un être d'action et d'instinct qui se définit par sa force physique. Homme du peuple, forgeron de sa profession et peu instruit des choses du monde, Zishe partira vers l'inconnu pour trouver son destin et accomplir la mission que Dieu lui assigne.

Dans les scènes à la ville, la quête d'identité et de connaissance de Zishe s'oriente vers des voies multiples qui prendront la forme de révélations. Deux scènes apparaissent déterminantes. L'une illustre la gloire de Zishe en héros germanique (9. "Siegfried the Iron King ») et l'autre décrit son contact avec la musique pure (13. "Princess Martha's Music »). Cet accès à la connaissance artistique se présente également sous la forme d'une vision qui fera l'objet d'une analyse. Un deuxième type de connaissance, associé lui aussi à une vision, prendra sens à travers le contexte historique de l'entre-deux-guerres. Parmi les trois scènes analysées dans la seconde partie figure celle du voyage de Zishe vers le nouveau monde (6. "Seeking a New World »), marquant le passage entre la campagne et la ville. La seconde scène est celle représentant Zishe en Siegfried. La troisième est celle où Zishe découvre la musique par l'entremise de Martha, la pianiste. Le choix de ces trois scènes a été dicté par l'importance que chacune d'elles accorde à la musique et à la représentation du héros germanique tiré de la mythologie wagnérienne. Aux fins de l'analyse proposée en seconde partie du texte, je suspendrai l'action d'Invincible pour traiter plus à fond des nombreuses dimensions du héros et de ses connotations wagnériennes.

\section{Zishe, le nouveau Siegfried}

J'entame ici une première analyse comparative entre le personnage principal d'Invincible et le héros wagnérien de La Tétralogie en vue de démontrer la présence wagnérienne dans le film par la représentation du héros. Seront évoqués des traits de personnalité, des actions symboliques et des éléments narratifs favorisant un tel rapprochement. Pensons à la scène où Zishe apparaît sous les traits de Siegfried. Il s'agit de la première prestation du héros devant le public allemand. Pour eux, Siegfried est l'incarnation par excellence de la race aryenne. Selon Wagner, Siegfried est le type idéal de l'humanité future, l'homme le plus parfait. Ce nouveau Siegfried accomplira devant eux un acte symbolique en courbant trois fois une épée de fer. Dans l'opéra Siegfried, troisième épisode de La Tétralogie, le héros apparaît comme un être ignorant

6 « Tu vas partir, aller dans le monde... Mais n'oublie jamais qui tu es ». 
la peur, qui seul réussira à reforger Notung, l'épée éclatée de Siegmund. L'épée revêt une dimension importante dans tous les opéras de Wagner : elle incarne la force indestructible et la domination du bien sur le mal. Dans La Tétralogie, elle se transmet de père en fils, dans Parsifal, elle guérit la plaie du roi Amfortas et assure la survivance d'une lignée royale élue. Dans notre histoire, Zishe n'a jamais employé son métier pour forger des épées ou des armes, mais plutôt des instruments d'utilité courante. Ainsi, la scène qui le représente en Siegfried ne le montre pas en train de forger l'épée, mais bien en train de la détruire en la pliant dans un acte de domination. De sorte que Zishe le forgeron, qui s'était toujours employé à créer, se retrouve ici à supprimer l'objet issu de la forge. Par ce geste, il met son identité en question et désacralise le pouvoir de l'épée, ce qui marque son opposition contre tout pouvoir instrumental et contre toute magie. Par conséquent, la scène, qui permet en un premier temps de comparer Zishe à un héros germanique, demeure dans son action en désaccord avec le métier de Zishe, alors que sur le plan symbolique, elle respecte son identité en marquant une prise de position. Il sera question plus loin de la manière dont la musique juxtaposée à cette scène contribue à l'émergence des deux dimensions. Ajoutons que, dans la même scène, Zishe détruit les chânes qui l'assaillent et montre en cela sa capacité à se délivrer seul de toute tentative pour le soumettre. Zishe se présente donc comme un être libre et sans peur.

Ce qui pousse le Siegfried de Wagner à partir à l'aventure est son ignorance de la crainte, ce phénomène que tous éprouvent, sauf lui, et qui agit telle une fascination. Il la découvrira lors de l'éveil de Brünnhilde dans un sentiment mêlé de désir et de peur. Quant à Zishe, homme du peuple, la crainte de la menace nazie le force à quitter la ville pour faire part de sa vision à son peuple et le protéger ${ }^{7}$. Or, au départ, lorsque Zishe quitte sa famille, il est guidé par une nécessité d'accomplissement, sans qu'il ne la comprenne exactement, contrairement à Siegfried qui agit selon des buts précis. Une deuxième dimension rapproche Zishe de Siegfried quant à la motivation première qui les force à partir en croisade, soit la recherche du père. Siegfried n'a connu ni mère ni père, ce qui explique sans doute son vitalisme et son reniement pour Mime, le gnome qui l'a élevé. Siegfried, après avoir reforgé l'épée de son père, part à la recherche de ses origines. Selon la thèse de Philippe Godefroid dans Le jeu de l'écorché (1986), cette recherche du père à travers l'Art témoignerait de la rencontre entre l'Homme et son Père céleste ${ }^{8}$. Cette thèse se situerait à la base du wagnérisme (Godefroid, dans Wagner 1826-68, 115). Toutefois, il faut entendre par Art l'union de l'Homme avec la nature, qui en retour agit à la manière de la nature selon sa nécessité : «L'œuvre d'art véritable est celle qui

7 La vision de Zishe est décrite dans le film par des images au fort potentiel symbolique dans le tableau 19 («Future Ministry of the Occult», 1.22.35). Lors d'un rêve, Zishe se voit debout parmi des milliers de crabes rouges menacés par le passage d'un train. Ces images pourraient faire référence à la multitude de Juifs exterminés ou déportés dans les camps de réfugiés. À la suite de ce songe prophétique, Zishe déclare au rabbin qu'il a reçu une mission, celle d'être le nouveau Samson de son peuple (23. " An Unknown Just Man »).

8 Cette idée exprimée par Philippe Godefroid dans Les opéras imaginaires de Wagner $(1826-68,115)$ résume la thèse de son livre précédent, Le jeu de l'écorché (1986). 
fait disparaître les dernières traces de la volonté créatrice, qui s'affranchit de la pensée dans l'action sensible, qui réconcilie la science et la vie » (Wagner $1849,65)$. De même que " la nature engendre sans intention selon le besoin " (59), le héros qui fait de sa vie une œuvre d'art le fait souvent par inconscience, comme lorsque Siegfried forge l'épée sans connaître le métier de forgeron. Ce mythe du savoir instinctif se rapporte à la croyance romantique du génie humain qui reproduit la nature sans même en connaître les lois. De même, le don de force de Zishe demeure inexpliqué et génial, car issu de la nature. C'est par ce don que le personnage de Zishe atteint la représentation de l'Art, c'est-à-dire en commettant des gestes qui revêtiront un sens précis par rapport à l'ensemble des actes qui définiront sa mission, sa raison d'exister. Ces gestes constitueront l'œuvre de Zishe sur terre sans que ce dernier n'en soupçonne l'aboutissement. De même, lorsque Zishe quitte son père et sa famille, la recherche du Père céleste est d'abord inconsciente chez lui, même s'il fut élevé dans la foi.

Dans le tableau 15, Zishe ose révéler sa véritable identité au public allemand : «I am not a germanic hero [...]. But a new Samson. A Jew. And I'm proud of it $^{9}$ » («A changed Samson », 1.07.44). Zishe devient immédiatement un héros pour la communauté juive berlinoise qui l'invite à se joindre à elle lors d'une célébration en son honneur. La scène du sermon du rabbin illustre bien le lien de Zishe au Père céleste et la confiance que Dieu donne à ses disciples :

I will lift up mine eyes unto the hills from whence cometh mine strength. My strength cometh from the Lord, which made heaven and earth. He will not suffer thy foot to be moved. He that keepeth thee will not slumber. Behold, He that keepeth Israël should neither slumber nor sleep ${ }^{10}$. (16. «Zishe Finds His Faith », 01.14.20)

Dans une autre scène, Samuel le rabbin relate la légende juive des 36 Justes choisis par Dieu pour porter les souffrances de l'humanité. Selon Samuel, Zishe est l'un de ces Justes. Le rôle de Rédempteur du peuple apparaît chez Zishe comme chez Siegfried, l'un étant relié au religieux et l'autre au monde magique. La destinée hautement symbolique des deux héros se lit à la fois chez Siegfried, pour qui la révélation du père agit comme moteur de l'action, et Zishe, par la révélation de sa foi et sa vision du peuple juif en danger. Par-dessus tout, le lien au père détermine le départ et le retour de Zishe vers les siens et se situe ici au cœur de la structure du film ${ }^{11}$.

9 « Je ne suis pas le héros germanique que vous croyez [...]. Place au nouveau Samson. Un Juif, et fier de l'être».

10 « Je lèverai mon regard vers les collines, là d'où provient toute ma force. Ma force vient du Seigneur, l'auteur du ciel et de la terre. Il ne laissera pas trébucher ton pied, lui qui t'a protégé, il ne somnolera pas. Voici que Dieu, lui qui a veillé sur Israël, ne somnolera pas, ni ne sommeillera. »Cette traduction est tirée de la version française du DVD. Il s'agit dans le film d'une adaptation du Psaume 120 du Livre des Psaumes sur le thème du " gardien d'Israël » (Sabourin 1988, 534).

11 La scène du sermon intervient au milieu du film pour renouer avec les origines juives du héros au sein du monde civilisé. Elle représente l'intégrité de Zishe où qu'il soit, comme dans la fable du coq. 


\section{LA MUSIQUE COMME SUPPORT DU MYTHE}

La musique originale du film, composée par Hans Zimmer et Klaus Badelt, ne constitue que 70,6 pour cent de la musique entendue dans le film. Considérée comme une musique de fosse, elle accompagne l'image de façon extra-diégétique ${ }^{12}$, c'est-à-dire à partir du hors champ (off), et est produite par des musiciens extérieurs à l'univers du film. Les autres musiques sont produites par des personnages. Il s'agit de musiques diégétiques qui proviennent du hors champ ou du champ (in), c'est-à-dire produites par des musiciens présents dans l'image ou des modes d'enregistrement intégrés dans l'univers du film. Parmi ces musiques d'écran figurent les chansons « Sweet and Lovely » et « You're the Cream in My Coffee » (par Max Raabe et le Palace Orchestra) interprétées par le chanteur et les musiciens du Palais de l'Occulte (Zimmer et Badelt 2002, pl. 12, 13), dans un style classique et élégant en vogue dans les années 1930. Ces musiques comptent pour 11,4 pour cent de la durée totale d'Invincible.

Passons à l'étude de la musique de fosse composée par Hans Zimmer et Klaus Badelt pour les besoins expressifs du film. Absente de la première partie du film où l'action se situe en Pologne, elle intervient au moment où Zishe quitte son village natal pour cheminer vers Berlin. Dès lors, la musique de fosse est entendue tout au long du film et reste présente dans les scènes finales lorsque Zishe revient dans son village. Les thèmes entendus précédemment lors des scènes à la ville sont alors réitérés. Ainsi réapparaît le thème de la scène du voyage vers Berlin lorsque le héros retourne à la campagne. De même, le thème associé à Siegfried survient lors des dernières scènes en Pologne, thème désormais associé à Samson. Ces extraits marquent l'aspect du voyage et le transfert du personnage d'un lieu à l'autre, tout en restituant le passé du héros motivant ses actions futures.

Mentionnons également la présence parmi la musique de fosse d'airs de Haendel étrangers à la musique originale. Ainsi, on entend l'air "Ohne trost, ohn'alles Hoffen » (« Nulle consolation si tous n'espèrent », acte 1) de l'opéra Giulio Cesare (1724) lors du générique du début en hors champ. À la suite de quelques prises de vues en mouvement (travellings), l'air entre dans le champ lorsque l'image montre le gramophone d'où provient la musique. D'abord associé aux images de la communauté juive, l'air sera entendu une deuxième fois dans les scènes à la ville, lors de la visite de la mère et du frère de Zishe à Berlin. Dans une scène extérieure où Zishe écrit à sa famille, l'air « Dank sei Dir, Herr » (« Remercie ton Seigneur $\left.{ }^{13} »\right)$ renvoie à la foi et aux valeurs du personnage. Ainsi, les airs

La force intérieure qui motive Zishe à partir dans le monde, alors qu'il ignore encore la nature de sa mission, prend ici la forme de la religion. Plus tard, lors d'une vision, la mission que Dieu réservait à Zishe lui sera révélée : sauver les siens.

12Selon Marc Vernet, dans Esthétique du film $(2001,81)$, la diégèse désigne « l'histoire comprise comme pseudo-monde, comme un univers fictif dont les éléments s'accordent pour former une globalité ». La diégèse regroupe en somme tous les éléments inhérents à l'univers du film et les dépasse : « [Elle] désigne l'histoire et ses pourtours, l'histoire et l'univers fictif qu'elle présuppose et qui lui est associé " (Vanoye et Goliot-Lété 2001, 31). En retour, tout ce qui est extra-diégétique est étranger au pseudomonde reproduit par le film, bien qu'il contribue de manière extérieure à la valeur esthétique du film. Une musique extra-diégétique ne reproduit pas une époque ou un lieu décrits par le film, mais commente l'action en y apportant un point de vue.

13Les airs de Haendel entendus dans le film sont interprétés par la soprano Emmi Leisner (Preiser Recordings, 1927). Selon le musicologue Martin Elste (2004), l'air « Dank sei Dir, Herr », prétendument 
de Haendel rappellent à la fois la Pologne natale du héros et fonctionnent comme des musiques faisant référence à l'identité juive. La musique vocale de Haendel, considérée par les Juifs comme une réussite du répertoire classique, a été intégrée à la culture de ce peuple, de sorte que certains airs de Haendel font partie du folklore ${ }^{14}$. On entendra également, lors de la scène du cinématographe, l'air célèbre « Ombra mai fu ${ }^{15}$ » de l'opéra Serse (Haendel 1738, acte 1). Par la texture sonore, on peut déduire que ces airs ont été captés par les moyens techniques des années 1920, ce qui renforce le lien entre la musique et l'époque. Autre élément important, les musiques d'écran, qui constituent le paysage musical des scènes à la campagne, deviendront des musiques de fosse à partir des scènes à la ville. Elles se mêleront ensuite à la musique originale dans la dernière partie du film située à la campagne, de manière à mettre en évidence la rencontre de ces deux univers musicaux dans l'expérience du héros et de signifier son appartenance à la culture juive et son cheminement spirituel. Alors que Zishe revient chez lui, il renoue avec ses origines, d'où la présence des airs de Haendel. Cependant, il porte en lui quelque chose qu'il n'avait pas à son départ pour la ville, soit un sens conféré à sa vie, que la musique a pour fonction d'évoquer.

Les exemples musicaux qui servent aux analyses suivantes sont des transcriptions de trois extraits tirés de la bande originale du film (Zimmer et Badelt 2002), soit : 1. «The Journey » (2.04), 2. «Siegfried the Iron King » (2.09) et 5. « Souls » (3.07). Seuls certains thèmes, motifs, accords et caractéristiques harmoniques seront mentionnés et reproduits afin de relever des éléments significatifs de la structure musicale relative aux images, à l'action et aux personnages du film. Ces analyses visent à comparer des fragments de la partition musicale de Zimmer et Badelt avec des éléments stylistiques et référentiels appartenant à d'autres musiques. En effet, la musique originale du film, écrite dans un style plutôt romantique, s'inspire non seulement d'opéras de Wagner, mais aussi d'œuvres de compositeurs postromantiques comme Gustav Mahler et Samuel Barber. Ces emprunts iront parfois jusqu'à la citation ${ }^{16}$. Toutefois, il s'agit d'une esthétique propre à la musique de film dans laquelle les allusions aux grands compositeurs de tradition classique demeurent d'usage courant. Ce qui différencie la musique de film composée par Zimmer de celle d'un Mahler, par exemple, est principalement l'orchestration, réduite au cinéma pour diminuer les coûts d'enregistrement avec orchestre symphonique ${ }^{17}$. En ce qui concerne la technique particulière de Hans

attribué à Haendel, serait plutôt une œuvre de Siegfried Ochs (1858-1929) publiée en 1905.

14Plusieurs oratorios écrits par Haendel portent sur des sujets juifs tirés de l'Ancien Testament et ont valu au compositeur une forte estime auprès de ce peuple. Parmi les oratorios les plus représentatifs figurent Esther (1732), Israel in Egypt (1739), Messiah (1742) et Judas Macchabeus (1747). Voir Lemaire 2001.

15Interprété par Emmi Leisner, enregistré en 1928 par Deutsche Grammophon.

16Par exemple, l'influence de l' «Adagietto » de la Symphonie $n^{\circ} 5$ de Mahler (1902) est palpable dans « Visions » (pl. 7, tabl. 19. «Future Ministry of the Occult»). Cet extrait fait également grand emploi de l'accord de Tristan (accord diminué avec tierce mineure renversée [Pazdro 1988, 301]). La dynamique et la texture polyphonique de "Visions " présentent également d'étonnantes similitudes avec l'Adagio for Strings de Samuel Barber (1936).

17 En réenregistrant certaines parties instrumentales, les créateurs de musique de film obtiennent une texture plus dense dont le résultat se confond souvent avec celui d'un orchestre symphonique. C'est ce qu'on appelle overdubbing en anglais. Dans le cas de Zimmer et Badelt, il s'agit de véritables 
Zimmer, et qui caractérise des compositeurs de Media Ventures dont fait partie Klaus Badelt ${ }^{18}$, elle consiste à utiliser des sons de synthétiseurs à l'intérieur d'une partition de style classique en y adjoignant des solos d'instruments acoustiques.

Hans Zimmer, né en 1957 en Allemagne, est aujourd'hui un compositeur très en vue à Hollywood. Ses débuts sur la scène pop rock européenne au sein des groupes Krisma et Ultravox lui permettent d'expérimenter les techniques numériques du synthétiseur qui serviront plus tard à l'élaboration de musiques de film. Sa musique pour Crimson Tide (1995, réal. Tony Scott) le propulse à Hollywood et le conduit à composer la musique de The Peacemaker (1997, réal. Mimi Leder) pour les studios Dreamworks dirigés par Steven Spielberg, desquels il sera nommé directeur musical en 1998. Après avoir remporté les Oscars de la meilleure musique originale pour Driving Miss Daisy (1989, réal. Bruce Beresford) et The Lion King (1994, réal. Roger Allers et Rob Minkoff), Zimmer reçoit sept nominations pour Gladiator (2000, réal. Ridley Scott) et trois nominations pour The Thin Red Line (1998, réal. Terrence Mallick), que les inconditionnels de Zimmer considèrent comme son dernier chef-d'œuvre à ce jour, et dans lequel il expérimente différents stimuli visuels et sonores. La collaboration avec Herzog est récente et unique dans la carrière du compositeur qui offre dans Invincible une partition de facture classique. Par la texture sombre des cordes et la tension dramatique qu'elle suggère, la musique d'Invincible rejoint celle de The Thin Red Line. Elle intègre souvent des éléments de la culture des personnages du film, par exemple le chant tribal dans The Lion King, le ukulélé dans Pearl Harbor (2001, réal. Michael Bay) et la guitare et la voix dans Gladiator $^{19}$.

Trois scènes déterminantes du cheminement et de l'identité mythique du héros serviront à l'analyse des relations entre les images et la musique visuelle de Zimmer. À une brève description de chaque scène succédera une discussion concernant l'orientation du discours musical relatif à l'image. Suivront des comparaisons entre le langage musical des compositeurs Hans Zimmer et Klaus Badelt, les harmonies wagnériennes et l'esthétique musicale postromantique. Les scènes analysées sont délimitées par leur inscription à l'intérieur d'une durée musicale contenue dans le film. La méthode d'analyse comparative et sémiotique repose principalement sur la théorie des tensions harmoniques de Deryck Cooke telle qu'exposée dans The Language of Music (1973).

\footnotetext{
instruments doublés par des synthétiseurs. Grâce à cette technique, le timbre caractéristique des instruments et l'intensité obtenue numériquement émulent l'orchestre, alors que le contenu harmonique demeure réduit. Enfin, il faut mentionner que les éléments transcrits lors des analyses subséquentes correspondent davantage aux sons perçus dans le film et qu'elles ne sont aucunement reproduites à partir des partitions originales.

18Hans Zimmer a cofondé, avec Jay Rifkin, les studios Media Ventures (Santa Monica, Californie) qui offrent la possibilité à des compositeurs de travailler dans des conditions optimales et qui permettent le partenariat.

19«Biography for Hans Zimmer », http://www.imdb.com/name/nm0001877/bio (consulté le 28 janvier 2005).
} 


\section{«Vers un nouveau monde »}

La scène «Vers un nouveau monde » marque le passage entre deux lieux du film, soit la campagne natale de Zishe et la ville de Berlin. Cet épisode du voyage confère une grande place à la musique et à l'image, et ne comporte aucun dialogue. La caméra suit pour l'essentiel le personnage de Zishe à travers la campagne rurale. Il s'agit d'un thème en $f a$ majeur entièrement confié aux cordes. Ce thème complexe au large ambitus, qui atteint rapidement les sommets et semble attiré vers les profondeurs, est annoncé à la contrebasse et accompagné aux cordes qui forment la majeure partie de l'orchestration. Comme le montre l'exemple 1, la contrebasse fait d'abord entendre la sensible, un $m i$ grave soutenu, suivi d'un contour mélodique ascendant sur l'accord de septième de sensible $(m i-s o l-s i$ bémol - ré) tenu par les altos et les violoncelles, où figure un emprunt au mode de $m i$ mineur.

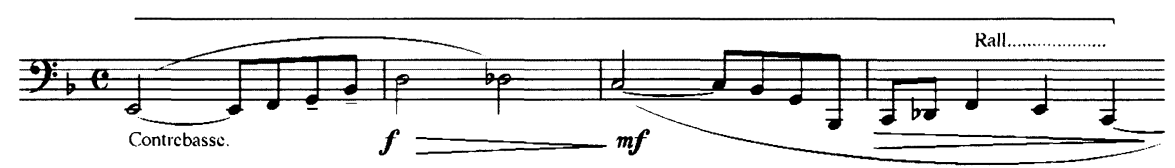

Exemple 1. Thème 1 (thème du destin), mes. 1-4

Le sommet du thème est atteint par un intervalle de tierce majeure qui donne lieu à une impulsion rythmique et mélodique ascendante. Ces éléments combinés créent un effet de tension et de détente dans lequel l'atteinte du sommet (ré) en valeurs longues déclenche aussitôt une lente désinence chromatique. L'instabilité ainsi produite préfigure une chute imminente que la musique réalise, tandis que l'image demeure fixe. Une telle incongruité entre le mouvement de l'image et le dynamisme mélodique du thème indique un lien expressif entre la musique et les mots prononcés ultérieurement par le père : « don't ever forget who you are! ». Ces paroles auront une incidence considérable sur le vécu du personnage et la suite des événements. Le décalage entre les paroles provenant de la scène précédente et la réponse musicale sur les images de la scène suivante accentue le lien entre le passé de Zishe et sa marche vers l'avenir.

Malgré les sommets du thème atteints par des tierces majeures ascendantes caractérisant un sentiment positif ( $s i$ bémol - ré, mes. 1-2; et ré bémol - fa, mes. 4), la présence du chromatisme et le tempo lent confèrent à la mélodie un caractère douloureux, près de la tristesse (Cooke 1973, 56). Au début du thème, l'accord de septième de sensible accentue également le caractère tragique propre au mode mineur. L'utilisation de croches (mes. 1 et 3 ) marque un empressement à atteindre un registre supérieur, puis à reprendre du grave un second mouvement ascendant (mes. 4). Cette partie conclusive du thème entendue en rallentando intervient de manière à résorber la rapidité et l'énergie des mouvements directionnels en croches par un contour flou et modéré en secondes et tierces, qui tend à revenir sur lui-même avant de se déposer dans les profondeurs du registre (mes. 4). 
L'accord de septième de sensible, qui annonce les couleurs du premier thème, est utilisé par Wagner dans le prélude de Tristan et Isolde pour préfigurer la fatalité du lien amoureux. Dans Invincible, cet accord est entendu harmoniquement aux violoncelles, sur le premier temps de la deuxième mesure $(m i-s o l$ - si bémol - ré). Le choix de faire entendre les notes de l'accord de manière mélodique et harmonique contribue à installer un climat de malédiction ou de mauvais présage qui se révèle dans l'accent provoqué par l'accord tenu aux cordes sur le sommet du thème et sa désinence ${ }^{20}$. L'impact violent de l'accord de septième de sensible sur les contours du thème est également signifié par les figures de note en rallentando. En effet, l'apparition de valeurs longues ( $r e ́$ - ré bémol $-d o$ ) crée un effet de suspension quasi statique, comme pour retarder l'inévitabilité du mouvement descendant.

Du mi grave sur lequel s'était déposé le thème 1 ( « thème du destin »), se dessine un motif ascendant qui atteint le registre médian de la partition (motif $a$, exemple 2). Ce dernier est similaire au motif conducteur du « Rhin » (exemple 3) (Flinois, dans Pazdro 1988, 763), tiré du premier acte de L'or $d u$ Rhin de Wagner et formé sur l'accord de mi bémol majeur.

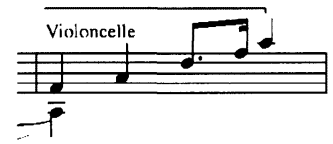

Exemple 2. Motif $a$

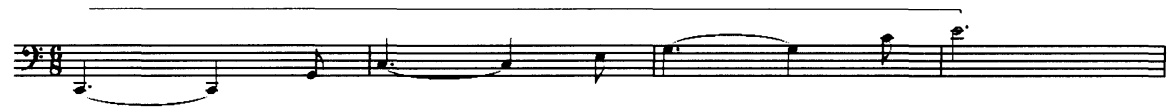

Exemple 3. Motif du « Rhin », tiré de L'or du Rhin de Wagner

Malgré les quelques différences d'intervalles (tierces et neuvièmes majeures et mineures), il existe bien une parenté mélodique entre les deux motifs qui se déploient sur un tempo andante. Leur position stratégique mérite également d'être soulignée. En effet, ces motifs marquent un commencement, soit le début de l'aventure initiatique de Zishe et les premières notes de La Tétralogie. Dans le motif $a$, la prégnance des tierces sur l'accord de $f a$ majeur, dont le ton est souvent associé à des sentiments simples et joyeux (Cooke 1973, 184), lui donne une dimension plus volontaire et hérö̈que que le motif du « Rhin », dont la portée symbolique (le commencement du monde) dépasse et absorbe l'héroïsme individuel. Ces deux motifs sont annoncés aux cordes. Cependant, le registre grave du motif du « Rhin » confirme une provenance obscure qui gravit un ambitus plus large. L'image des profondeurs du Rhin et celles qui dépeignent Zishe gravitant les collines de campagne avec le fleuve en aval illustrent

$20 \mathrm{~J}$ 'ai choisi de ne pas reproduire l'accord harmonique dans l'exemple 1 pour des raisons d'espace. D'autre part, je considère que cet accord ne fait pas réellement partie du thème, mais en accentue le contenu harmonique. Il est difficile de savoir si, dans la partition d'Invincible, cet accord a été créé à partir d'un enregistrement de l'instrument ou reproduit par synthétiseur à partir d'un seul enregistrement en changeant la hauteur des sons. 
cette différence. D'autre part, on remarquera que l'utilisation de tierces dans le ton de $f a$ majeur est souvent associée au caractère pastoral chez Beethoven (l'exemple de la Symphonie pastorale dans Cooke 1973, 184). De plus, l'emploi de notes arpégées de l'accord majeur dans un mouvement ascendant accentue souvent le caractère énergique et héroïque d'une mélodie (Cooke 1973, 228).

Le motif $a$ agit également à titre de motif annonciateur du héros, à la manière de la fanfare de cuivres qui scande les apparitions de Siegfried dans le Ring (exemple 4) (Flinois, dans Pazdro 1988, 772), mais plus subtilement. Parallèlement, le motif $a$ agit tel l'appel de cor dans Don Juan (op. 20) de Richard Strauss (exemple 5), qui signifie la venue du héros par une mélodie ascendante sur un large ambitus.

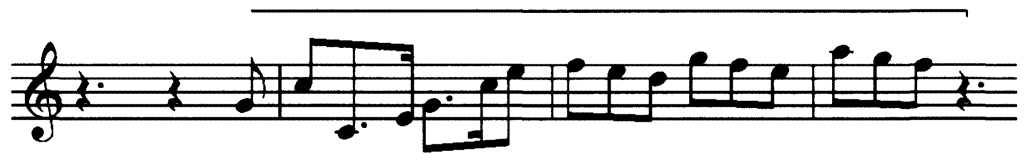

Exemple 4. Fanfare de Siegfried, un appel de cor tiré de Siegfried de Wagner

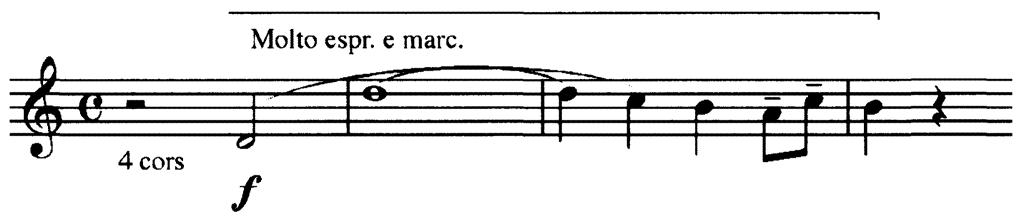

Exemple 5. Appel de cor, tiré de Don Juan (1889) de Richard Strauss

Ce motif entamé sur l'octave rend le changement de registre immédiat. À la différence du motif $a$, qui se déploie de manière mélodique en faisant entendre les notes de l'arpège, l'appel de cor s'affirme avec violence, à cause de sa compression mélodique. En conséquence, le motif $a$ annonce un héros bien différent, qui évolue selon la nature qui l'entoure en respectant la loi des harmoniques sur laquelle repose la notion d'accord. Les images du héros parmi la nature en éveil (lever du jour) confirment cette symbiose. Toutefois, l'importance secondaire du motif $a$ au sein de la partition musicale le limite à une simple fonction d'énonciation ou de préambule à la venue du héros. Il est entendu en simultanéité avec l'entrée du soleil dans l'image et le mouvement de caméra qui tranche avec l'immobilisme de la scène précédente. Il accompagne le héros suivi de profil, qui apparaît désormais nimbé de lumière. Le motif marque donc le passage entre l'aurore et le jour et la marche du héros vers sa destinée.

Tout en ponctuant l'entrée du motif $a$, les violons débutent une série de figures diatoniques en doubles croches procédant par intervalles de quartes et quintes, créant ainsi un effet de scintillement imitant le mouvement ondulatoire de la lumière sur le fleuve perçu en aval. Ce procédé de composition est utilisé par Wagner dans le prélude de L'or du Rhin («Entrée des dieux dans le 
Walhalla ») aux cordes. Les secondes et les tierces superposées sur l'accord majeur de sol bémol y sont accompagnées par des arpèges à la harpe dans une sorte de démultiplication rythmique et mélodique (exemple 6).

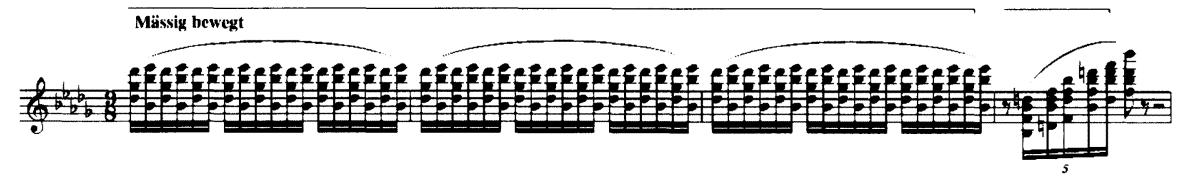

Exemple 6. «Entrée des dieux dans le Walhalla », extrait de L'or du Rhin de Wagner

Remarquons également, parmi les similarités entre le prélude du Rhin et l'extrait musical choisi, l'annonce de l'arrivée des dieux aux cordes et aux vents formant un motif arpégé ascendant (reproduit à l'exemple 7), semblable au motif $a$.

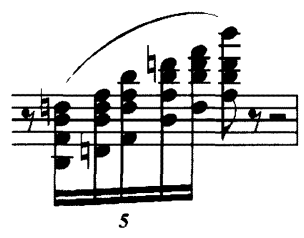

Exemple 7. «Entrée des dieux dans le Walhalla », extrait de L'or du Rhin de Wagner

L'appel conduit, dans les deux cas, du grave au registre médian où s'amorce le thème principal (dans l'ouverture, le motif du « Rhin »). Le prélude renvoie à la genèse du Ring et aux dieux qui émergent des abysses d'un fleuve magique, « réservoir infini de toutes les possibilités de l'existence » (Stéphane Goldet, dans Pazdro 1988, 708). Parallèlement, le chemin de Zishe vers un nouveau monde coïncide avec la première apparition de la musique originale du film. Avant cet extrait, les musiques entendues étaient essentiellement des musiques d'écran dans un style populaire. L'entrée de Zishe dans le monde lui permet également d'atteindre, par un tel accompagnement musical et sa résonance rhénane, la représentation du héros germanique dans les opéras de Wagner.

Les motifs d'introduction analysés préparent l'entrée du thème principal d'où seront extraits les motifs subséquents du développement musical. Un deuxième thème se déploie avec ampleur aux violons à partir de la dominante, do (exemple 8).

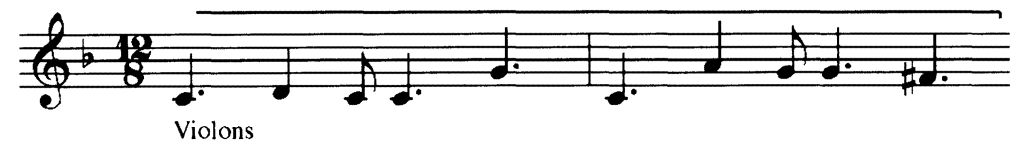

Exemple 8. Thème 2

Des intervalles de quintes et sixtes majeures interviennent de manière ascendante et rejoignent rapidement les aigus. La sixte ascendante, souvent utilisée dans l'opéra (avec les septièmes) pour signifier les épanchements passionnés 
ou les élans du cœur, caractérise le motif du « désir » dans Tristan et Isolde (exemple 9) (Capacci, dans Pazdro 1988, 321).

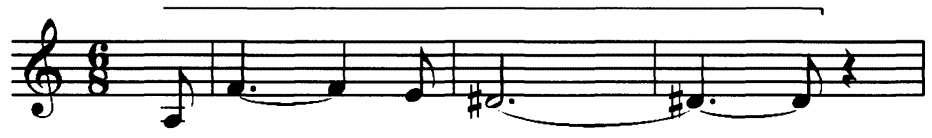

Exemple 9. Motif du « désir », dans Tristan et Isolde de Wagner

Dans les deux exemples, la sixte mineure est suivie de secondes. On remarquera également que le thème et le motif ne reviennent pas sur la tonique comme l'atteste la fin du thème 2 sur sol et $f a$ dièse. Ce traitement mélodique de la sixte ascendante réussit à en maintenir l'élan et l'effet expressif. De même, en évitant un retour à la tonique, le contour descendant empêche de reproduire mélodiquement l'effet inverse de sixte descendante, par exemple dans le motif du « philtre de mort » de Tristan (do - ré dièse) (exemple 10) (Capacci, dans Pazdro 1988, 321). Dans cet exemple, la sixte descendante signifie l'écroulement, la mort. Elle s'oppose en cela au sentiment positif du désir, donc à une avancée vers l'avant : elle est régression.

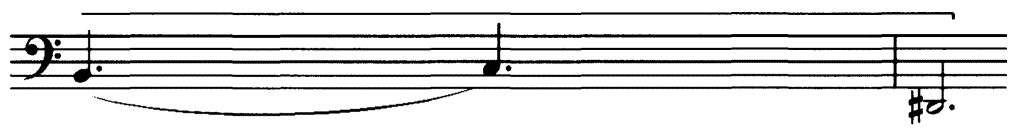

Exemple 10. Motif du «philtre de mort », tiré de Tristan et Isolde de Wagner

Les images montrent un vaste paysage jalonné de champs et de rivières où le personnage, toujours à l'avant-plan, poursuit sa marche. Le champ apparaît de plus en plus vaste grâce au recul de la caméra par rapport au personnage. Les larges intervalles du thème, dont l'intervalle de sixte, suggèrent la grande étendue ainsi que le relief ascendant des collines au sommet desquelles se dresse le héros. Dans ce genre de musique romantique à caractère pastoral, la sixte ascendante du thème 2 devient l'évocation du vertige ou d'une émotion liée à l'excitation, ce qui accompagne efficacement les vues en plongée sur la vaste campagne allemande. Les sentiments associés à la sixte proviennent du caractère ouvert de cet intervalle. De plus, l'attirance de la sixte (la) vers la dominante $(\mathrm{sol})$ permet au thème de prendre ses distances face à la tonique et de faire flotter la mélodie au-dessus de son pôle tonal (Cooke 1973, 69). De plus, la résolution du thème sur la quinte correspond à un fondu enchaîné sur image et un motif ascendant en triolets à la clarinette.

La clarinette intervient de manière ponctuelle sous forme de dialogue avec les violons, sans former de mélodie maîtresse. Son utilisation n'est pas fortuite, puisqu'elle figure parmi les instruments mélodiques en premier plan dans la musique Klezmer, associée à la culture juive. La présence de cet instrument dans des extraits musicaux subséquents simultanés à l'apparition de Zishe permet de supposer que leur association vise à souligner l'origine juive du personnage. Toutefois, l'emploi de la clarinette demeure purement classique 
et se confond avec le lyrisme des cordes, ce qui dément cette hypothèse. Or, dans une scène suivante où Zishe traverse une plaine, on entend une musique d'écran où la clarinette évoque les origines populaires de la culture juive. Des paysans, venus à la rencontre du nouveau Samson dont la réputation leur est parvenue, jouent du violon et de la clarinette. L'extrait marque l'entrée de Zishe dans la légende juive. Quant à l'emploi classique de la clarinette, il symboliserait la marche de Zishe vers la civilisation urbaine, dont les scènes avec musique sont caractérisées par l'élément orchestral. La clarinette, associée à la culture du héros, conjugue l'origine et la quête de Zishe à un univers musical romantique de type wagnérien.

La séquence suivante montre Zishe endormi sous un pommier alors que le thème module en ré mineur. Les intervalles ascendants correspondent au réveil de Zishe et à la poursuite de son périple. Intervient ensuite une série de figures en croches et triolets formés de secondes et tierces aux altos et aux vents qui mettent en valeur les contours du thème en allégeant la texture sonore. Le tout accompagne une nature en éveil. Chaque nouveau développement motivique est issu du thème 2 , dont il partage les principaux traits mélodiques : triple répétition de la note de base du thème et enchaînement par imitation de la tête ou de la fin du thème précédent à travers un processus de variations.

Une sixte mineure descendante accompagnée de figures descendantes aux cordes marque le passage à un second motif varié dans un épisode modulant de ré majeur vers la tonalité de sol bémol majeur (motif $b$, exemple 11).

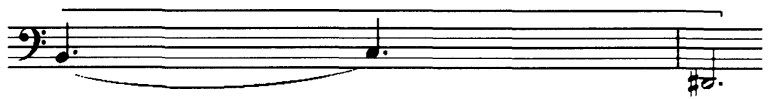

\section{Exemple 11. Motif $b$}

Les changements de ton coïncident avec un second fondu enchaîné sur l'image en plan éloigné de deux fermiers aux prises avec une charrette enlisée. Un second plan montre Zishe allant vers eux pour leur offrir son aide. En musique, cela correspond au même passage modulant caractérisé par une insistance répétée sur $f a$ dièse ( $\mathrm{sol}$ bémol) qui suppose une modulation au triton. Le motif de base évolue peu en hauteur et en mouvement, à la manière de Zishe qui concentre son propre poids avant de soulever la charrette. Il repose sur une dynamique d'effort et d'appui illustrée par l'alternance de noires et de croches. Cette figure rythmique semble tout indiquée pour évoquer l'effort physique. En effet, Wagner utilise cette combinaison rythmique en valeurs diminuées pour son motif du « travail » (exemple 12) (Flinois, dans Pazdro 1988, 773).

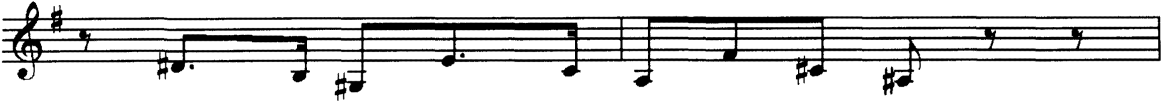

Exemple 12. Motif du «travail », extrait de Siegfried de Wagner 
Toutefois, l'aspect disjoint et mobile du motif du « travail » ne correspond pas au type d'effort fourni par Zishe, dont la force s'accumule de manière progressive et repose sur une position stable et concentrée. Par conséquent, le motif $b$ s'arrête immédiatement lorsque la charrette est soulevée. Dans cet épisode, le cinquième degré du ton suivant, ré bémol (do dièse dans le motif $b$, exemple 11) est enharmonique. Enfin, toute l'action du soulèvement de la charrette est accompagnée par un passage modulant qui signifie un changement de transport pour le personnage.

Tout au long de la première partie en do majeur et en ré mineur, les cordes et la clarinette forment des figures indépendantes en valeurs diminuées, ce qui donne une vivacité et un aspect fluide à l'orchestration. Les motifs ont plusieurs fonctions : amortir les contours descendants de la mélodie, accentuer ou remplir un intervalle ascendant sur une valeur longue. Les figures arpégées occupent aussi la fonction de remplir les longues tenues de la mélodie, par exemple $f a$ dièse lors du passage modulant, et de varier le caractère répétitif de ce passage. Elles opposent un caractère interrompu et concis à la ligne continue de la mélodie. Les figures en $f a$ majeur annoncent déjà de manière imbriquée l'entrée dans le nouveau ton de sol bémol majeur. Ainsi, comme à chaque début de nouvelle partie, les cordes gagnent les profondeurs du registre pour mieux accentuer l'ascension vers un nouvel élément musical.

\section{«Siegfried, le roi de fer »}

C'est lors de la scène intitulée "Siegfried, le roi de fer » que s'effectue l'association de Zishe à Siegfried. L'action se passe à Berlin dans le luxueux Palais de l'Occulte d'Hanussen, où le public assiste à la première prestation de Zishe sur scène. Le présentateur, annonçant les exploits relevés par Zishe, relate la légende ancienne de Siegfried combattant avec les Cimbres et les Teutons lors de l'invasion de la Gaule en 102 av. J.-C. : fait prisonnier par les Romains, Siegfried est enchaîné, mais sa force le délivrera de ses liens. Puis, il arrivera à plier l'épée de son ennemi. Ces deux actions se produiront sur la scène devant un public allemand acclamant leur nouveau Siegfried. La partition musicale majoritairement confiée aux cordes fait également intervenir le cor et des sonorités électroniques.

Les premières notes se font entendre en pianissimo aux contrebasses. Siegfried (Zishe) est montré de dos, vu des coulisses. Fidèle à l'iconographie des héros germaniques, il porte le heaume et le carquois, et arbore une perruque blonde pour créer une fausse origine aryenne. Un la grave tenu sur six temps fait place à un aussi long si accompagné d'un motif rapide aux cors sur la seconde ré - $m i$, tel un tremblement. Le timbre particulier des cordes graves et la faible intensité évoquent une force lointaine qui émerge lentement. Ces premières manifestations sonores sont accompagnées de sons étouffés imitant le battement du cœur. Le son, traité en réverbération, semble reproduit électroniquement en séquences court-long, ce qui donne à la texture sonore un aspect corporel et une tension palpable. Le tout se déroule dans un tempo lent, comme une respiration soutenue et profonde. L'introduction ponctue le passage à un second plan fixe, 
cette fois sur le public constitué majoritairement de généraux et de soldats nazis, subjugués devant la vision du nouveau Siegfried. La basse se fait également entendre sur les mêmes notes à l'octave inférieure. Superposées, les trois lignes qui oscillent sur les mêmes notes forment un crescendo élaboré sur différentes valeurs de notes : rondes, blanches pointées, noires, doubles-croches. L'intervalle de seconde ( $l a-s i)$ ainsi multiplié crée un sentiment d'instabilité, de fébrilité : le risque encouru par Zishe est grand. Lorsque ce dernier déchire le tissu qui le recouvre, le public se lève aussitôt en applaudissant. Finalement, un ré s'impose à l'alto pendant six temps, alors qu'un nouveau plan en plongée montre des officiers évaluant les chaînes qui serviront à la prochaine démonstration de force, plan au cours duquel débute le motif principal. Concentré autour de la tonique, le motif $c$ (exemple 13) évolue par blanches de manière conjointe et diatonique. L'intensité croît à mesure que le motif se répète. Il se déploie lentement comme s'il transportait avec lui une charge. Le ré tenu en ostinato par les cordes graves accentue la lourdeur du motif, constituée par l'attirance vers la tonique et les intervalles conjoints qui le maintiennent dans le registre médian.

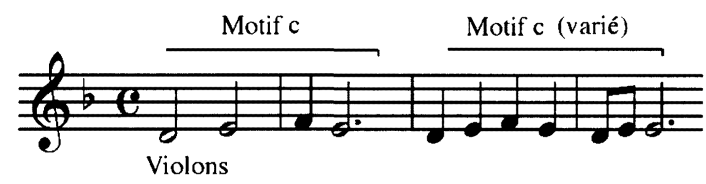

Exemple 13. Motifs $c$ et $c$ varié

Il s'agit maintenant de la scène où Siegfried est enchaîné. Dès que le héros rompt les chaînes qui entourent son buste, le motif se fait entendre en noires avec une insistance sur $m i$ (motif $c$ varié, exemple 13). Le $m i$ est gardé plus longtemps à la fin du motif et s'impose à la suite du ré comme un nouveau pilier. Plus tard, lorsque Siegfried tord l'épée, le motif se dépose sur sol et se développe vers un degré supérieur par plateaux à mesure que le héros accomplit un nouvel exploit. Le motif $c$ est alors transposé sur $f a(f a-s o l-l a-s o l)$. Lorsque Siegfried brise ses chaînes, une note tenue au cor se fait entendre en crescendo, puis diminue d'intensité sur $d o$ (septième degré en ré mineur). Cette intervention du cor crée un malaise par sa proximité avec le ré et le mi entendus dans le motif précédent (motif $c$ varié) et son attirance naturelle vers la tonique. La tension entre la septième et la tonique sera résorbée par la transposition du motif $c$ varié sur $f a$, simultanément avec le cor qui intervient alors sur l'intervalle de dominante (motif sur $f a$ et cor sur $d o$ ). À ce moment, la musique atteint un sommet d'intensité. L'affirmation du cor à travers le matériau mélodique aux cordes exprime une empathie pour le personnage de Zishe, dont on ressent l'effort et la concentration. Concurrente à la gloire qui salue les exploits de Zishe, la tension, amorcée par l'intervention du cor, de même que son affirmation au sein de la mélodie, persiste jusqu'à la fin de la représentation.

L'intensité, la durée, les effets spéciaux auxquels sont soumis les solos d'instruments et les liens entre la musique et l'image confèrent au timbre une dimension référentielle. Ainsi, les interventions du cor sur les images d'une 
foule de nazis en train d'applaudir et d'acclamer accroissent la portée dramatique de la scène tout en soulignant le sentiment de danger inscrit dans ce soulèvement. Dans un tel contexte, la résonance stridente et soutenue du cor peut faire penser aux sirènes utilisées par le régime fasciste durant les années 1930, qui semaient la peur parmi les communautés juives. Ces sirènes étaient utilisées pour prévenir les résidents d'un quartier ou d'un établissement de l'arrivée de la police ou des troupes nazies.

Revenons à la description de la scène. Les plans sur le public montrent des attitudes inquiètes et une attention soutenue. Lorsque les membres du public se lèvent une deuxième fois pour l'applaudir, Zishe se sent soutenu dans son épreuve. De même, le motif principal associé à la force apparaît aux cordes graves en parallèle à la tierce inférieure. Ce support mélodique a pour effet d'amortir la tension produite par la transposition du motif sur $f a$, soit à la tierce mineure supérieure. Un second motif formé d'une sixte mineure et d'une seconde majeure descendante apparaît dès la fin des applaudissements du public pour signifier la fin de l'ascension de Zishe. Si le motif précédent décrivait une lente montée en force, celui-ci, par l'utilisation d'un intervalle large et descendant, signifie une chute de tension. De plus, il apparaît comme l'inversion du thème initial lors de la scène du voyage (thème 1), pour en boucler l'évolution en quelque sorte. La présence du motif descendant associé au thème $1 \mathrm{du}$ premier extrait musical se met en parallèle avec le destin de Siegfried dans La Tétralogie. Ainsi, il symbolise la mort du héros au moment même de son ascension vers la gloire. Face au triomphe de Zishe, la musique continue de se faire menaçante ou de maintenir un climat émotionnel tragique. Ce deuxième motif décrit ainsi l'effondrement imminent du personnage, alors que l'image montre Zishe brandissant l'épée qu'il va bientôt tordre. Ce mouvement musical en simultanéité avec les images montrant Zishe courbant l'épée crée une distance entre la musique et l'image, qu'il est possible d'associer à la mise en danger de Zishe contenue dans sa consécration, mais aussi au fait qu'il renie par ces gestes sa propre identité en revêtant celle de Siegfried.

Durant cette scène, un second appel de cors retentit de la même manière que le premier, mais cette fois sur sol, quatrième degré en ré mineur. La tension créée dans cet extrait par les interventions du cor provient de la confrontation entre deux tonalités voisines, do et sol formant mélodiquement un rapport I - V. Ces deux notes, dont la première rappelle le ton initial du premier extrait musical ( Vers un nouveau monde »), indiquent à la fois une continuité et une rupture entre les deux extraits. Par le passage à la dominante, elles rappellent le ton d'une situation initiale et introduisent mélodiquement une perturbation. En outre, le choix du cor n'est pas anodin dans l'orchestration de cet extrait, puisqu'il est associé, dans La Tétralogie, au Rhin et aux apparitions de Siegfried. Cette dimension ténébreuse et dissonante de l'utilisation des cuivres dans l'extrait contribue à substituer de manière autoritaire, par le premier et le cinquième degré d'un autre ton, le personnage de Siegfried à celui de Zishe. Lorsque le cor intervient au cinquième degré, le nouveau ton est établi. Il s'agit donc du ton de Siegfried, puisqu'il apparaît au cor, instrument qui accompagne toutes les apparitions de Siegfried dans les opéras de Wagner. Ainsi, cette 
intervention du cor comme symbole de Siegfried montre qu'il y a bel et bien eu pénétration du personnage wagnérien à travers Zishe. Cela induit une transformation du héros initial qui, en faisant symbiose avec la figure de Siegfried, revêt une importance symbolique en tant qu'homme idéalisé par une nation ${ }^{21}$. D'autre part, en se montrant à la face du monde en Siegfried, Zishe renonce à une partie déterminante de son identité et la dissimule à ses détracteurs. À la fin de l'extrait, le rappel du motif $b$ a déjà agi comme motif conducteur et ramène à un passé proche. Cette dernière apparition du motif ne se résout point sur la tonique, mais bien sur le deuxième degré, la laissant ainsi suspendue. Seule la basse se dépose sur ré (tonique) à la suite d'une appoggiature longue sur mi. Ce type de résolution imparfaite suppose que la démonstration de force de Zishe ne sera pas la dernière.

\section{« Princesse Martha et la musique »}

Après une session d'hypnose devant le public, Hanussen réussit à prouver que la force du corps peut rivaliser avec celle de l'esprit. La force de Zishe devient en quelque sorte le moyen pour Hanussen de démontrer la supériorité de la race allemande dans sa perfection physique et spirituelle. Lors d'une seconde séance d'hypnose, Hanussen prononce une apologie de la puissance allemande et se nomme le prophète de l'avènement du Führer. Pour Hanussen, le discours idéologique et la construction des mythes font partie du spectacle : il s'agit de donner au public ce qu'il demande. Consécutivement à Hanussen, un autre personnage important du Palais de l'Occulte sera révélé. Zishe, ayant entendu une musique provenant de la salle de spectacle, fait la connaissance de Martha.

Si la scène analysée précédemment reposait sur la comparaison entre la figure de Zishe et celle de Siegfried, la scène dont il sera maintenant question porte sur une autre dimension androgyne du personnage de Zishe ${ }^{22}$. Cette dimension correspond à l'accès de Zishe à la connaissance artistique par sa relation avec le personnage de Martha comme incarnation de la musique pure. Dans l'optique des écrits wagnériens, Siegfried, le héros masculin aux possibilités supérieures, représenterait le Poète, soit l'artiste de l'avenir qui, telle la nature, agit par nécessité. De même, selon L'œuvre d'art de l'avenir, le poète et le peuple qui reconnaissent leur détresse commune s'uniront dans l'œuvre d'art (Wagner 1849, 68). La scène permettra de constater que la conception de la musique pure de Herzog cohabite avec celle que Wagner évoque dans ses écrits théoriques. Quant à la question de l'androgynie, elle est sous-tendue dans l'opéra Siegfried par l'union du héros avec Brünnhilde (fin du dernier acte). Ces unions révèlent une essence salvatrice. Dans le cas d'Invincible, l'union du féminin et du masculin repose sur une compréhension mutuelle par l'inter-

21 Le personnage de Siegfried fait partie de plusieurs contes et légendes remontant aux origines du peuple allemand. Il symbolise par sa force et sa constitution physique l'homme « parfait et pur » de la race germanique. Il s'oppose en cela à la figure du Juif allemand, soit à une " race » prétendument « impure ».

22Pour l'aspect androgyne chez Wagner, voir Nattiez (1990). Ici, l'androgynie du personnage masculin se rapporte à son union symbolique avec le personnage féminin et non à des traits physiques ou caractériels. Ensemble, la force naturelle et la musique s'unissent dans l'idée d'authenticité. 
médiaire de l'Art symbolisé par la vision de Martha. En raison de la rencontre des deux personnages, entre autres, l'analyse musicale de cette scène reposera davantage sur des dimensions harmoniques et thématiques, et moins sur le contenu mélodique qui, dans les scènes précédentes, caractérisait davantage l'aspect solitaire du héros.

Dans cette scène, les plans de caméra sont peu importants. En majorité fixes, ils effectuent parfois de légers travellings de manière à suivre les personnages. Les dialogues entre Zishe et Martha sont montés en plans parallèles, ce qui sied à une dynamique interlocuteur (Zishe) / narrateur (Martha). À la suite d'une demande de Zishe, qui a entendu Martha au piano, celle-ci relate le récit de son passé et de ses études au Conservatoire de Prague. Au cours du récit, les violons et les altos effectuent une lente montée diatonique dans le ton de si majeur (thème 3 , exemple 14).

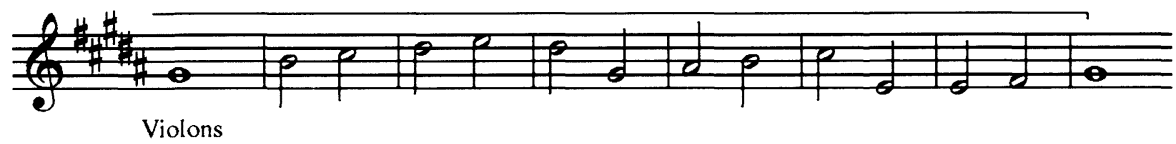

Exemple 14. Thème 3, mes. 1-8

Suspendue sur $m i$ à la troisième mesure, et retombant sur ré dièse à la mesure suivante, la gamme ascendante est ensuite reprise à la quinte inférieure (sol dièse). Elle se dépose enfin sur la sixte majeure (do dièse - mi) à la sixième mesure. Comme dans le cas des premier et deuxième extraits, la mélodie ascendante se déploie sur une durée plus longue que les intervalles descendants, qui ont pour fonction de la transposer afin qu'elle poursuive sa montée tout en demeurant dans le même registre. Ce procédé de composition permet d'inscrire le mouvement musical au sein d'un ambitus réduit. Le registre défini permet, s'il n'est pas dépassé, de circonscrire les aspirations et les questionnements humains qui caractérisent ces scènes de dialogue et de les maintenir à l'échelle humaine, à la manière de chaque motif évoluant au sein de la tonalité.

Le troisième thème utilise, comme le thème 2 de "Seeking for a New World ", les intervalles de quinte et de sixte. Toutefois, la quinte (ré - sol, mes. 4) et la sixte ( $d o-m i$, mes. 6) sont descendantes, soit davantage associées à la mort ou à l'action de céder (voir motif du « philtre de mort », exemple 10). Quant au thème 4 (exemple 15), il utilise les mêmes intervalles de seconde que le thème 2 , mais de manière inversée, dans un contour descendant. Il existe donc plusieurs parentés mélodiques entre les thèmes 3 et 4 (associés à Martha) qui semblent issus du thème 2 (associé au personnage de Zishe).

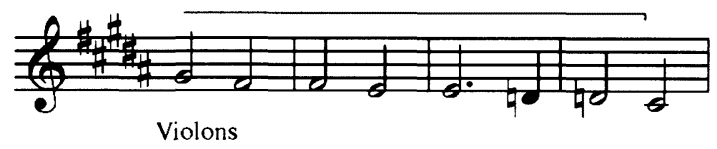

Exemple 15. Thème 4, mes. 1-4 
Parmi les distinctions entre ces thèmes, le thème 2 tend davantage vers le registre aigu et possède un ambitus plus large. Cette différence marque bien la distinction entre le personnage de Zishe et celui de Martha. D'une part, les intervalles issus du thème de Zishe reviennent systématiquement à la tonique, alors que, dans le thème de Martha, leur fonction est principalement de déplacer le modèle d'un point focal à l'autre. Autrement dit, l'attirance vers la tonique agit davantage pour caractériser Zishe, alors que le thème de Martha évolue de manière quasi indépendante au sein de la tonalité, comme dans un état de flottement. De même, une tonique appuyée caractérise la force physique qui accomplit des exploits à partir de son propre poids et doit compter sur une structure solide, affirmée. De plus, une tonalité diffuse permet de donner une importance accrue à la mélodie, soit aux relations horizontales entre les sons, en créant toutefois un effet de suspension, tout à fait dans l'esprit du langage musical expressionniste d'un Mahler ou d'un Schoenberg.

La scène, qui personnifie Martha, renvoie principalement aux choses de l'esprit, d'abord par l'évocation du passé, puis par celle du rêve, c'est-à-dire le désir de Martha de jouer avec l'orchestre le Concerto $n^{\circ} 3$ en do mineur, opus 37 de Beethoven. Une musique légère et ascensionnelle correspond justement à ce type de sentiment associé à la rêverie et à l'aspiration. La scène révèle également les statuts orphelin et apatride de Martha, qui la définissent en tant qu'être détaché ou abandonné du monde. Ainsi, malgré le caractère tonal de la musique qui l'accompagne, les pôles tonaux sont évités.

La suite du thème de Martha présente une gamme par tons effectuant une lente désinence (exemple 15). Le passage procède par appoggiatures dont chacune marque un pallier nouveau. Cette utilisation de l' appoggiature rappelle beaucoup le traitement mélodique de l'" Adagietto » de la Symphonie $n^{o} 5$ de Mahler, qui s'impose également avec lenteur et grâce (exemple 16).

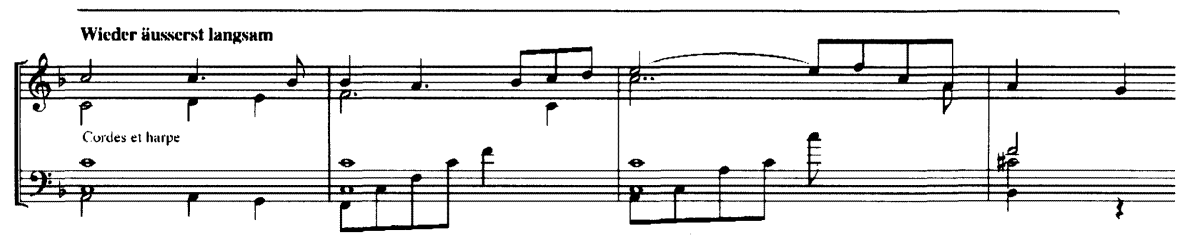

Exemple 16. «Adagietto », $4^{\mathrm{e}}$ mouvement de la Symphonie $n^{\circ} 5$ de Mahler, mes. 23-26

À ce moment, la musique souligne les mots de Martha portant sur la rencontre avec Hanussen qui fit obstacle à ses aspirations artistiques. Pour commenter l'événement raconté, la clarinette effectue une descente en secondes et tierces sur l'accord mineur de si. Après s'être déposé sur $d o$ bécarre, le thème gagne un registre plus grave sur l'accord de la majeur dont la neuvième ( $s i)$ constitue la tonique. De $s i$, les cordes graves remontent à sol dièse, alors que la clarinette se fait entendre à nouveau, cette fois dans un contour ascendant sur les notes de l'accord majeur du IVe degré ( $m i)$. Chaque accord du passage, que l'on croirait modulant, contient toujours la tonique du ton principal; les accords annoncent toutefois la venue d'un cinquième thème. Dans cet extrait (« Princess Martha's Music »), la musique souligne les moindres épanchements 
affectifs de Martha nourris par la réminiscence de souvenirs passés. Il s'agit sans doute de la page musicale où le procédé de la Durchkomponiert est le mieux illustré dans sa qualité descriptive.

À la fin du dialogue entre Zishe et Martha, cette dernière dévoile sa vision. C'est alors que les violons qui soutenaient la mélodie reviennent pour faire entendre le thème de Zishe tiré du premier extrait musical, lequel apparaît maintenant transposé à la septième majeure supérieure, ce qui correspond au registre du thème de Martha. Les cordes graves répètent simultanément le thème descendant de Martha en guise de contrepoint, alors que s'y mêle celui de Zishe. Les images montrent ensuite un rideau fermé que Zishe et Martha ouvriront ensemble. Transposé une deuxième fois à la septième inférieure, le thème de Zishe rejoint le registre médian, se rapprochant davantage de celui de Martha. Les deux personnages pénètrent dans une salle ronde dont les murs vitrés révèlent un aquarium. Ils contemplent avec fascination les méduses blanches qui évoluent gracieusement, tandis que les violons reprennent le thème descendant de Martha. Celui-ci, qui soulignait d'abord le caractère soumis de Martha, intervient à deux reprises à la voix supérieure. Il est réitéré sur l'image des méduses captives derrière les murs en verre du salon d'hypnose d'Hanussen. À la lumière des apparitions du thème descendant, formons l'hypothèse qu'il convient à la description d'âmes pures, en l'occurrence celles de Martha et des méduses subissant l'emprise d'un personnage dominant, Hanussen. Ici, l'idée de pureté est essentiellement induite par l'allusion à la musique pure contenue dans le discours de Martha.

Des motifs secondaires apparaissent aux cordes en guise de contrepoint pour soutenir le passage lent et gracieux, tout à fait à l'image du mouvement des méduses. Aux deux voix qui se développent harmoniquement, mais de manière presque indépendante, s'ajoute une note tenue aux cors effectuant un crescendo, puis un diminuendo. L'accent ainsi produit rappelle la présence du cor dans le deuxième extrait musical. Associé désormais à Zishe, le cor ajoute à la texture musicale en gravitation une troisième voix harmonique, sans susciter de tension. En introduisant cette troisième voix dans la composition qui présente différents niveaux mélodiques, le cor amplifie l'impression de densité et de profondeur associée à la mer. Le passage suggère que le tout mélodique baigne dans une harmonie quasi parfaite. Sur les mots de Zishe adressés à Martha, « Now, I see your soul ${ }^{23} »(1.01 .52)$, deux figures ascendantes émergent tour à tour du registre grave jusqu'au registre médian. Elles se basent essentiellement sur un accord diminué arpégé ( $\mathrm{fa}-\mathrm{la}$ bémol $-d o$ bémol - $m i$ bémol) dont la parenté avec l'" accord de Tristan » est évidente : il s'agit de permuter la tierce (la bémol) à l'octave supérieure pour l'obtenir : $f a-d o$ bémol $(s i)-m i$ bémol (ré dièse) - la bémol. Cet accord spécifique se trouve à osciller entre le ton de $m i$ bémol majeur et celui de sol bémol mineur. Le motif final de l'extrait apparaît à deux reprises, en se déposant sur sol bémol (tonique de l'accord de VII ${ }^{\mathrm{e}}$ diminuée) en appoggiature, puis sur $s o l$ bécarre. La finale sur les notes $s o l$ et $s i$ bémol dessine un accord incomplet qui entraîne, comme pour l'accord de Tristan, une ambiguïté harmoni-

23 « Maintenant je vois votre âme ». 
que, de sorte qu'il devient impossible de savoir à quel ton appartient l'accord. La réponse se trouve toutefois suggérée par le rapprochement musical et thématique entre le couple Tristan et Isolde uni dans l'amour, et celui de Zishe et Martha uni dans l'art. Comme ces fusions ne se distinguent pas l'une de l'autre, l'accord incomplet sol - si bémol recèle bien deux tonalités, l'une majeure et l'autre mineure, autant dire le masculin et le féminin.

L'extrait multiplie les correspondances entre la conception de la musique suggérée par Herzog et la théorie de la musique pure chez Wagner. Les images montrant des créatures marines se mouvant dans l'élément liquide préfigurent la musique dans son état le plus pur nageant dans la "mer de l'harmonie » (Wagner 1849, 120). La filiation de ces créatures avec la musique prend tout son sens dans les paroles prononcées par Martha : « Now look at these beings. For me, they have the purest of souls. I have never seen anything more beautiful [...]. I want to play music to match this vision ${ }^{24}$ » (13. «Princess Martha's Music » : 01.12.19). Toutefois, les écrits de Wagner rappellent que la musique pure ne pourra se changer en mélodie qu'à la condition d'une union avec ses deux sœurs, la poésie et la danse. Ainsi, le mouvement des méduses symbolise dans le film la musique en mouvement, c'est-à-dire alliée à la danse. En ce qui concerne la poésie, tout porte à croire qu'elle s'incarnerait dans la figure de Zishe. En effet, seul ce dernier permettra à la musique de se libérer de la mer de l'harmonie, car le passage de l'harmonie à la mélodie doit être fécondé par le langage des mots, soit le langage poétique (Nattiez 1990, 98).

Une telle hypothèse vient justifier la présence de l'appel de cors dans la texture harmonique, de même que l'identification de Zishe à Siegfried. Dès le son du cor entendu lors du deuxième extrait musical, Zishe entre déjà dans la légende des héros wagnériens. Ainsi, il continue d'y être associé durant toute la période qui se déroule à Berlin. Par le transfert Zishe/Siegfried, le premier entre dans l'univers mythique wagnérien. Comme dans les opéras de Wagner, le héros représente ici le point central du déroulement dramatique. Peu importe en réalité le chemin qu'il aura à parcourir, il passera toujours d'un état de connaissance à un autre, et ce, jusqu'à sa chute (Godefroid, dans Wagner $1826-68,511)$. En cela, il agit comme le poète qui donne l'exemple au peuple. Enfin, la citation suivante, tirée d'Opéra et drame, ne saurait mieux résumer cette force extérieure qui pousse Zishe vers le monde et permet son accès à la connaissance artistique par l'entremise de la musique :

Partant de la note fondamentale de l'harmonie, la musique s'était étendue sur une surface énormément variée, où finalement elle s'était mise à la disposition du musicien absolu qui nageait sans trêve ni but : il ne voyait rien devant lui qu'une houle infinie de possibilités, mais en lui-même, il n'avait conscience d'aucun but déterminant ses possibilités (Wagner 1850b, 154-55).

La musique, sous sa forme harmonique, agit en quelque sorte comme le parangon des possibilités ouvertes à l'homme qui, à l'image de la nature, se

24 « Regardez ces créatures! Pour moi, leurs âmes sont d'une pureté parfaite. Je n'ai jamais rien vu d'aussi beau [...]. Je veux que la musique que je joue soit comme cette vision ». 
meut selon ses besoins ou sa nécessité intérieure. Les dernières lignes sont les plus significatives à cet égard par rapport à Zishe. Car, malgré sa conviction de posséder des qualités supérieures qui lui permettront d'aller plus loin, le véritable but de son existence et la nature de ce qu'il devra accomplir lui demeurent inconnus. L'image des méduses, coïncidant avec le retour du « thème du destin » relatif au voyage, prouve par association que Zishe trouve une réponse à son questionnement dans la vision de l'harmonie. Dans L'œuvre d'art de l'avenir, Wagner $(1849,126)$ définit l'harmonie comme étant une force naturelle perceptible, mais non intelligible à l'homme. De cette manière, l'harmonie symbolise l'avenir sur lequel l'homme n'a aucune prise. Ainsi, Zishe n'a pu résister à l'appel du destin et à son attirance vers le monde.

Cette révélation de la musique s'accompagne donc d'une réflexion sur le mystère que constitue en soi la notion d'avenir. L'union de Zishe et de Martha étant symbolisée par la musique pure, notamment par la présence du cor au sein du thème de Martha, elle se réalise à travers l'exécution du Concerto $n^{o} 3$ de Beethoven ${ }^{25}$, symbole de leur union éternelle dans l'Art. Rappelons que, pour Wagner, la musique de Beethoven renvoie à l'union parfaite entre la poésie et la musique symbolisant l'homme et la femme (Nattiez 1990, 63). Beethoven, qui se nommait lui-même le «poète des sons » (Tondichter), incarne pour Wagner l'être humain complet qui, par sa nécessité de musicien, « se jette dans les bras du poète pour accomplir l'acte de génération de la mélodie véritable et rédemptrice " (Wagner 1850a, 185). Pour Wagner, une telle fusion de la poésie et de la musique se réalise parfaitement dans le dernier mouvement de la Symphonie $n^{\circ} 9$ de Beethoven, grâce à la présence du poème L'ode à la joie de Schiller. D'autre part, l'œuvre symbolise davantage la musique à programme que la musique pure, laquelle se retrouve plutôt dans la forme du Concerto $n^{o} 3$. Alors que celui-ci connote la victoire de la musique pure, tout porte à croire qu'il s'agit plutôt d'un sacrifice que d'une union, puisque la musique prend ici le pas sur la poésie. En effet, Zishe, qui incarne la figure du poète, n'accomplit qu'une mission intermédiaire, celle de libérer la musique pure. Le réel destin de Zishe est reporté à plus tard, dans un second sacrifice, non pas pour la musique, mais pour son peuple.

La légende de Zishe Breitbart se termine sur une note tragique, digne des opéras où les héros vont tout droit vers l'agonie. De retour en Pologne, Zishe tentera d'avertir sa communauté du danger provenant du régime fasciste allemand, alors que celle-ci, ignorant la réalité de la ville, se moquera de lui. Pour rallier quelques paysans sceptiques à sa cause, Zishe devra prouver sa force en enfonçant de sa main un clou rouillé dans une planche de bois. Sort du destin, le clou atteindra sa jambe, et la gangrène en nécessitera l'amputation. Rappelons que Siegfried lui-même, réputé invincible, meurt par une épée enfoncée dans le dos, son seul point vulnérable. Zishe, quant à lui, périt de

25Le deuxième mouvement du Concerto pour piano en do mineur de Beethoven est interprété dans le film par la comédienne incarnant Martha, Anna Gourari, avec l'Orchestre de la Beethovenhalle de Bonn. 
n'avoir pu convaincre ses frères du danger. Il succombe à plusieurs opérations deux jours avant la prise de pouvoir de Hitler.

Les analyses musicales précédentes, liées à l'argument du film, contribuent à élucider les rapports entre les images, les dialogues et la musique, et à comparer le personnage de Zishe au héros germanique, tout en éclairant la nature des liens qu'il entretient avec les autres personnages. Elles montrent comment la musique occupe un rôle primordial dans la définition des liens mythiques entre Zishe, Siegfried et la musique. Toutefois, parallèlement aux aspects révélés par la musique, les images et le discours fournissent des indices précieux sur la manière d'interpréter la musique originale du film. En effet, sans ce support des images et du texte, nulle comparaison ni association ne tiendraient entre la musique et le contenu pour attester l'influence qu' y exerce la mythologie wagnérienne. Ces considérations sur les liens entre le cinéma et la musique permettent donc d'avancer l'hypothèse que le film Invincible fonctionne à la manière des opéras de Wagner, c'est-à-dire en faisant appel à une union des arts afin d'ériger l'ensemble de l'œuvre. C'est pourquoi les propos suivants aborderont plus à fond les liens qui existent entre le film et l'opéra.

\section{PRÉSENCE DE L'OPÉRA DANS INVINCIBLE}

Le cinéma peut être défini comme un genre hybride qui allie écriture, images en mouvement et musique, sur fond de réalisme cinématographique. Comme l'opéra, le cinéma dépend des autres arts et en réalise la synthèse. Il s'oriente principalement autour de la reconstitution d'une image vraisemblable, que la musique doit servir (Ishaghpour 1995, 33). Cette image cinématographique se présente en retour comme la partie d'un tout ayant pour référent le réel. L'opéra, qui recourt également au pouvoir de l'image, vise la création d'une « image totale du monde ", c'est-à-dire d'un tout artificiel, entièrement reconstitué par l'art et dont l'ensemble est englobé par la musique. Comme le mentionne Ishaghpour $(1995,33)$, « [l']opéra se veut la réalisation du concept de l'art : la création d'une image totale du monde rempli de sens; du monde devenu musique, et de la musique devenue monde ». Le sens que remplit le monde de l'opéra est celui que donnent à l'action le héros et sa quête : c'est Siegfried qui libère l'homme du règne des dieux ou Zishe qui recherche l'authenticité dans une société aveuglée par ses mythes.

Comme il en a été question, la première scène intitulée "Seeking a New World » se présente comme un prélude aux aventures du héros. Comportant peu de paroles, hormis les quelques mots prononcés en introduction par le père du héros, ces séquences mettent de l'avant l'image et la musique. Celle-ci a pour rôle de transmettre au spectateur les émotions du héros, soit la fusion avec la nature et la confiance ressentie devant l'inconnu. Sans la musique nécessaire à cette scène, la marche du héros dans la campagne n'a de sens que pour le regardant qui y appose sa subjectivité et ne présente rien de plus qu'un passage géographique obligé en rapport avec le scénario. C'est précisément lorsque l'image agit seule, soit sans le texte, qu'elle nécessite la musique. Celle-ci agit en retour comme supplément à l'image en y ajoutant une signification profonde (Derrida, cité dans Kramer 1996, 
$111-12)^{26}$ : elle révèle ce nouveau monde entrevu par Zishe derrière les collines, par-delà un univers de possibilités nouvelles qui dépassent la simple apparence des choses et se situent au-delà de l'image. L'avenir vers lequel tend le héros par son attitude et son action apparaît ainsi comme une métaphore de la musique, c'est-à-dire comme une forme en devenir constant, mue par un sentiment. Comme en ce qui concerne l'opéra, la musique, vecteur d'émotion, donne sens à l'image. Elle recrée en cela la dimension de l'opéra au cinéma.

Il existe dans tous les opéras un thème central qui dicte l'évolution des personnages au cœur de l'action mythique et qui peut être comparé à la place qu'occupe le drame dans les opéras wagnériens. Il s'agit du thème de la fatalité qui supprime l'action relative au discours et fait place aux sentiments des personnages exprimés par la musique (Ishaghpour 1995, 31-32). En effet, le héros dans l'opéra agit toujours selon une logique temporelle préétablie propre au mythe, de sorte qu'il se trouve à subir le sort plutôt qu'à l'influencer. Hormis quelques actions symboliques, le héros réagit essentiellement par le chant en exprimant ses états d'âme face aux événements sur lesquels, malgré sa volonté, il n'a pas d'emprise. Par son dénouement et la présence d'événements historiques réels, le film Invincible porte également le sceau de la fatalité qui, selon Ishaghpour (1995, 33), correspond dans l'opéra à « l'exaltation musicale d'une salvation utopique ». Ce sentiment irréversible s'exprime dès le prélude («Seeking a New World ») par le thème du destin qui, par ses abrupts contours descendants, évoque la lutte vaine du héros. Au cours de l'action filmique, comme dans les opéras de Wagner, les personnages subiront les aléas d'une fatalité caractérisée par le déclin d'un monde et la nécessité de préserver ce qui peut être sauvé. Le thème se centre autour du sentiment d'une pureté perdue et prend appui sur le statut impur du monde représenté par la société industrielle et civilisée, c'est-à-dire le Berlin des années 1930.

Quant à la scène "Siegfried the Iron King ", elle recèle des éléments visuels empruntés à l' aspect théâtral de l'opéra. Les clichés contribuent à l'évocation du grand style opératique du XIX ${ }^{\mathrm{e}}$ siècle et à sa renommée comme art de l'ostentation. Tous les attributs de l'image, dont le palais luxueux, la richesse voyante de l'auditoire, la présence de l'élément scénique et le recours aux costumes, visent à recréer le lieu et la magie des grands opéras, tout en donnant libre cours aux actions du héros et au pouvoir de la musique. Cette dernière ouvre la voie aux sentiments de Zishe pris ici comme acteur et vecteur de l'action, puisqu'il joue le rôle de Siegfried jusqu'à un certain point. Les images témoignent également de la démesure du héros et de la force émotive de la situation, due à la présence d'un Juif parmi des Allemands. De même, comme à l'opéra où les auditeurs sont conquis par la prestation vocale des chanteurs, le public dans le film est subjugué par la force physique du protagoniste.

26L'idée de supplément est empruntée au philosophe Jacques Derrida, selon qui le supplément ne s'ajoute que pour remplacer ou combler un vide : «Qu'il ajoute ou qu'il substitue, le supplément est extérieur, hors de la positivité à laquelle il se surajoute, étranger à ce qui, pour être par lui remplacé, doit être autre que lui » (Derrida 1967, 208). Ainsi en est-il du relais de l'image par la musique. La musique intervient comme une addition extérieure. 
Autrement dit, la force agit ici comme le chant dans l'opéra en tant que « voix de la nature dans l'homme » (Ishaghpour 1995, 28). De plus, comme toujours à l'opéra, la musique exprime la tragédie intérieure du personnage. Dans l'exemple précédent, la concentration sur l'acte à accomplir et la peur de faillir devant le public allemand ennemi et adorateur révèlent cette part de vulnérabilité enfouie dans le personnage de Zishe.

La musique, loin d'exprimer une victoire acquise, soutient ce tour de force autant physique que symbolique et fait découvrir à l'auditeur la réalité affective du héros. En devenant elle-même acte héroïque, elle affirme le rôle central de celui-ci dans l'espace cinématographique. C'est à ce moment d'extrême intensité sonore que survient un climax musical qui excède la narration et remplit l'image entière. Les émotions vécues par le héros et transmises par la musique orientent en conséquence le regard du spectateur sur l'action. Comme dans l'opéra, la musique se substitue au temps et à l'espace du spectateur en se campant dans l'affect du personnage pour mieux amplifier la dimension épique du mythe et ainsi l'englober.

Le montage en parallèle du cinéma permet ici de jeter un regard sur les coulisses, la scène et le public, de manière à distinguer les moyens du spectacle et l'illusion produite sur le public. Ce dernier, susceptible de prendre le faux pour le réel, confond mythe et vérité. La musique originale, par sa qualité extra-diégétique, permet au spectateur extérieur de comprendre l'enjeu du héros, alors que le public représenté n'accède pas à cette dimension : il voit Siegfried tandis que nous entrevoyons Zishe. En faisant apercevoir autant les moyens que le résultat d'un processus intrinsèque à l'univers du film, le cinéma effectue une mise en abîme de la représentation. Par conséquent, alors que l'adhésion du spectateur extérieur à la réalité de l'image cinématographique devient totale, la thèse de l'opéra se trouve disqualifiée dans l'espace diégétique du film : l'opéra, associé au spectacle, renvoie une image fausse de la réalité.

La mise en abîme est utilisée dans ce cas pour signifier la présence dans l'image cinématographique d'une autre réalité construite sur les apparences : celle du spectacle. Feignant la réalité, le spectacle est ainsi représenté à l'intérieur du monde fictif du cinéma, lui-même une reconfiguration du réel. D'ailleurs, la caméra, qui montre à plusieurs reprises le point de vue des coulisses, soit l'envers du décor, renvoie elle-même à la fausseté de l'image. De sorte que cet envers du décor, qui prend pour objet le monde du spectacle tel que pensé à l'intérieur de la diégèse du film, c'est-à-dire la réalité des personnages, joue le rôle du réel objectif. S'ajoute aussi une réalité autre, également fictive : la représentation du héros mythique. Cette deuxième en abîme suggère la présentation d'un mythe, la légende de Zishe Breitbart, à l'intérieur d'un autre mythe, le retour du nouveau Siegfried. S'opposent ainsi l'histoire racontée par le film Invincible, soit le récit vécu d'un Juif, et celle surfaite de l'action filmique, soit la création d'un faux mythe : le retour de Siegfried, en vérité un Juif, comme modèle de la race aryenne.

La situation décrite dans cette scène centrale d'Invincible cultive une illusion propre à l'opéra, et surtout aux opéras de Wagner, qui considère le mythe comme révélateur d'une vérité. Cette croyance était répandue chez certains 
Allemands à l'époque où se situe le film. Alors qu'ils admettent la force surhumaine de Siegfried, ils ne peuvent concevoir et accepter celle de Zishe, un Juif. Lors des scènes d'idolâtrie qui précèdent le dévoilement de la réalité, c'est le mythe qui justifie la nature et non l'inverse. En d'autres termes, les Allemands présents dans cette scène adulent une image dans la mesure où elle les renvoie à eux-mêmes. Par des scènes montrant Zishe en tant que rédempteur de la race allemande, Herzog cherche à démontrer que la croyance et l'adhésion de l'auditoire pour l'action reposent uniquement sur l'image. En montrant un héros juif servant d'icône au nazisme, il défait un fondement essentiel de la religion juive basé sur l'interdit des images. Associée à l'idolâtrie, l'image trahit la pensée, le message : tout n'est qu'illusion. De même en va-t-il pour l'opéra qui ne capitalise ni sur le message ni sur un quelconque raisonnement, mais bien sur l'effet. Autrement dit, il vise l'emportement par le sensible en s'alliant l'irrationalité, l'aspect magique des choses et leur impact affectif sur le spectateur : il cherche à confondre la vie et le mythe.

\section{CONCLUSION}

Par-delà le cadre initial de l'essai, soit l'analyse de la musique et de la représentation du héros dans Invincible, la conclusion permettra de commenter une idée centrale propre au film étudié, soit la dichotomie entre Allemands et Juifs dans l'Allemagne des années 1930. Avec le recul, le regard porté par le cinéaste allemand sur ce pan d'histoire suppose une prise de position nouvelle et une réflexion prétendument plus objective sur ces événements. Ayant débuté sa carrière cinématographique dans les années 1970, Herzog fait partie des premiers cinéastes à aborder ce sujet délicat. Il est de la même génération que Rainer W. Fassbinder (Le mariage de Maria Braun, 1978; Effi Briest, 1974) et Volker Schlöndorff (Les désarrois de l'élève Torless, 1966; Le Tambour, 1979), dont les films interrogent la littérature allemande afin d'identifier les causes qui ont pu mener l'Allemagne à l'impasse nazie (Bellan 2001, 78-79).

Par un retour à une époque directement antérieure à la Seconde Guerre, Herzog a réussi à représenter, par l'entremise du monde du spectacle, la montée du sentiment haineux contre les Juifs, simultanément à la fascination des Berlinois pour les sciences occultes et les mythes germaniques. Pour couronner le tout, Herzog choisit un héros juif comme porteur de l'idéal aryen et se trouve à disqualifier la théorie nazie de la « race pure ". Cette figure de l'idéal se trouve alors renversée à travers son antinomie : une race soi-disant «faible » à éliminer. De plus, tous les personnages importants d'Invincible sont juifs, même celui que l'on soupçonne le moins de l'être, Hanussen ${ }^{27}$. Pourtant, un

27Erik von Hanussen est en vérité un nom emprunté à un noble danois. Hanussen, qui se nomme en vérité Herschel Steinschneider, est Juif. Cette identité est révélée lors de la scène du jugement (21. «Exposing Hanussen »). Seront également révélés, par Zishe et Martha Fahrah, les truquages utilisés lors des spectacles d'hypnose. Le personnage de Martha, né en Tchécoslovaquie, pose problème à l'égard de l'affirmation de l'identité juive. Toutefois, étant donné l'absence de territoire proprement juif comme condition d'une véritable nation, le statut de ce peuple se rapproche du statut apatride de Martha. Il existe également en Tchécoslovaquie une importante diaspora juive. 
trait de personnalité associé de manière caricaturale aux Juifs, soit un don légendaire pour l'argent et les affaires, se trouve justement dépeint par ce personnage, mis également en rapport avec le monde du spectacle.

D'autre part, Herzog prend soin d'inscrire son nouveau Siegfried à l'intérieur d'un récit mythique avec renfort de connotations wagnériennes, notamment sur le plan de l'aspect théâtral et du symbolisme conféré à la musique. La trame narrative comporte également des références à la religion et aux légendes juives dans lesquelles s'inscrit le personnage principal et qui constituent l'objet central du judaïsme (Ehrenfreund 2000, 5). Ces sermons et ces mythes au cœur des traditions et de la culture judaïques fonctionnent tout à fait à la manière des récits wagnériens, soit pour rappeler au peuple l'histoire de ses origines. Enfin, le parallèle entre la religion juive et les mythes allemands contribue à raviver la question d'une ambiguïté identitaire.

Dans Mémoire juive et nationalité allemande, Jacques Ehrenfreund (2000, 10) insiste sur cet élément au centre de la vie juive en Allemagne et démontre que « les cultures juives et allemandes [sont] constituées de métissages et de transferts culturels ». Dans Invincible, le fait que le héros passant pour Siegfried soit en vérité un Juif coïncide également avec la dimension universaliste de la pensée judaïque qui affirme « la centralité de l'histoire juive sur celle de l'humanité » (177). Moritz Lazarus (1900), auteur de Was Heist und $z u$ welchem Ende studirt man jüdische Geschichte und Litteratur? (Que signifie l'histoire universelle et à quelle fin l'étudie-t-on?), reflète cette mentalité juive :

Il est impossible de comprendre et d'apprécier l'histoire d'un peuple particulier sans l'envisager dans son rapport à l'histoire universelle et surtout pas l'histoire des peuples cultivés sans l'histoire juive, c'est-à-dire sans reconnaître l'influence exercée par le judaïsme sur tout le développement culturel ultérieur ${ }^{28}$.

Cette réflexion renvoie dans le film au questionnement du rôle de la culture juive dans la société allemande berlinoise. Toutefois, elle n'implique nullement que les Juifs soient au centre de l'histoire. Or, l'empreinte de cette mentalité explique qu'une importante communauté juive berlinoise ait continué, malgré la pression allemande, de se considérer elle-même comme une entité fondatrice de l'identité culturelle allemande.

Il existe également au sein de l'univers wagnérien une ambiguïté identitaire qui s'inscrit au cœur de la recherche artistique du compositeur. Celle-ci concerne Wagner lui-même et la question du véritable père, qui demeure encore aujourd'hui non résolue et qui induit la possibilité que Wagner soit de descendance juive. Cependant, même au niveau de l'étymologie des noms allemands et juifs, la parenté linguistique est telle qu'il est souvent difficile de les distinguer. Enfin, l'impression générale est celle d'un malentendu en ce qui 
concerne l'identité des Allemands et qui ravive une réalité fort complexe, soit celle de la fusion centenaire des deux peuples.

Je terminerai cette digression sur la question juive allemande sans fournir de véritable réponse, mais en citant toutefois un passage du film Invincible, dont l'importance s'avère maintenant centrale. Dans le tableau 10 («Hanussen, Master Clairvoyant » 0.51.42), Hanussen déclare : "What the power of the body can achieve, $[\ldots]$ the power of the spirit can also achieve ${ }^{29} »$. Par ces paroles, Hanussen n'adopte aucun parti, ne serait-ce que pour disqualifier le corps sur l'âme dans la démonstration prophétique qui suit. De même, il réfère à la fois au pouvoir du corps et de la pensée humaine, toutes nationalités confondues. Par ailleurs, cette phrase à caractère universel renvoie plutôt à l'équilibre entre la force physique et la force spirituelle de l'homme, et correspond davantage à l'adage de la civilisation grecque du « corps sain dans un esprit sain ». Cette phrase résume à elle seule la motivation commune des deux peuples qui, en fouillant leur passé, se rapprochent toujours plus d'une quête pour appréhender l'origine de leur identité, loin de tout syncrétisme culturel, soit d'une époque où les peuples allemands et juifs vivaient séparément, mais en harmonie.

\section{RÉFÉRENCES}

Barber, Samuel. 1936. Adagio for Strings. New York : Schirmer, Inc.

Bellan, Monika. 2001. 100 ans de cinéma allemand. Paris : Ellipse.

Cooke, Deryck. 1973. The Language of Music. Oxford : Oxford University Press.

Derrida, Jacques. 1967. «Ce dangereux supplément ». Dans De la grammatologie, 207-19. Paris : Les Éditions de Minuit.

Ebert, Roger. 2002. "Invincible ». Chicago Sun Times, 4 octobre. http://roger ebert.suntimes.com/apps/pbcs.dll/article?AID=/20021004/REVIEWS/2 10040301/1023 (consulté le 16 janvier 2005).

Ehrenfreund, Jacques. 2000. Mémoire juive et nationalité allemande : Les Juifs berlinois à la Belle Époque. Coll. "Perspectives germaniques». Paris : Presses universitaires de France.

Elste, Martin. 2004. «Ochs, Siegfried ». Grove Music Online, sous la dir. de Laura Macey. http://www.grovemusic.com (consulté le 13 février 2005).

Godefroid, Philippe. 1986. Le jeu de l'écorché : Dramaturgie wagnérienne. Paris : Papiers.

Haendel, George Friedrich. 1724. Julius Caesar (Giulio Cesare) : Opera in Three Acts, trad. angl. de Humphrey Procter-Gregg. New York : International Music, 1973.

—_ 1738. Xerses oder Der Verliebte König (Serse) : Heitere oper in drei akten. New York : C. F. Peters Corp., 1924.

29 « Ce que la puissance du corps peut accomplir, [...] la puissance de l'esprit peut l'accomplir aussi ». 
Herzog, Werner, réal. 2001. Invincible. Alliance Atlantic and New Line Home Entertainment. 2003. DVD.

Ishaghpour, Youssef. 1995. Opéra et théâtre dans le cinéma d'aujourd'hui. Paris : La Différence.

Kramer, Lawrence. 1996. Classical Music and Postmodern Knowledge. Berkeley : University of California Press.

La Rochelle, Réal. 2003. Opérascope : Le film-opéra en Amérique. Montréal : Tryptique.

Lazarus, Moritz. 1900. Was Heist und zu welchem Ende studirt man jüdische Geschichte und Litteratur? Ein Vortag. Leipzig.

Lemaire, Frans C. 2001. Le destin juif et la musique: Trois mille ans d'histoire. Paris : Fayard.

Lyon-Caen, Gilles. 2002. "Invincible de Werner Herzogr [sic] ». Objectif cinéma, rubrique "Point de vue ». http://www.objectif-cinema.com/ pointsdevue/0290.php (consulté le 16 janvier 2005).

Mahler, Gustav. 1902. Symphonie no. 5 für grosses Orchester : Adagietto. Leipzig : C. F. Peters, 1964.

Nattiez, Jean-Jacques. 1990. Wagner androgyne : Essai sur l'interprétation. Paris : Christian Bourgois Éditeur.

Pazdro, Michel, dir. 1988. Guide des opéras de Wagner. Paris : Fayard.

Sabourin, Leopold. 1988. Le livre des Psaumes traduit et interprété. Recherches nouvelles, série 18. Montréal : Bellarmin.

Strauss, Richard. 1889. Don Juan, Tone Poem for Full Orchestra, op. 20. New York : C. F. Peters Corp., 1982.

Sullivan, Moira. 2002. « Herzog's Invincible ». Movie Magazine International, 2 octobre. http://www.schoestring.org/mmi_revs/herzog'sinvinci-ms88172979 (consulté le 27 janvier 2005).

Vanoye, Francis, et Anne Goliot-Lété. 2001. Précis d'analyse filmique. Paris : Nathan.

Vernet, Marc, et al. 2001. "Récit, narration, diégèse ». Dans Esthétique du film, $3^{\mathrm{e}}$ éd., 75-85. Paris : Nathan.

Wagner, Richard. 1849. L'œuvre d'art de l'avenir. Dans Euvres en prose 3 : 59-254, trad. de 1'all. par Jacques-Gabriel Prod'homme et Fritz Holl. Paris : Librairie Ch. Delagrave, 1928.

- 1850a. Euvres en prose : tome IV : Opéra et drame I, trad. de l'all. par Jacques-Gabriel Prod'homme. Coll. «Les Introuvables ». Paris : Éditions d'aujourd'hui, 1982.

- $1850 \mathrm{~b}$. Euvres en prose : tome V: Opéra et drame II, trad. de l'all. par Jacques-Gabriel Prod'homme. Coll. «Les Introuvables ». Paris : Éditions d'aujourd'hui, 1982.

—_. 1826-68. Richard Wagner. Les opéras imaginaires, livrets d'opéras inachevés, trad. de l'all. et analysés par Philippe Godefroid. Paris : Librairie Séguier Archimbaud, 1989.

Zimmer, Hans, et Klaus Badelt. 2002. Invincible's Soundtrack. Milan Records, CD 198 736-2. 


\title{
Résumé
}

Dans son film Invincible (2001), le réalisateur allemand Werner Herzog met en scène la légende de Zishe Breibart, un Juif doté d'une force surhumaine. La théâtralité des images filmées par Herzog et la musique originale du film composée par Hans Zimmer concourent, selon l'auteure, à accentuer la dimension héroïque du personnage et à évoquer l'opéra wagnérien. Après avoir mis en parallèle les procédés de composition de la musique du film et de l'écriture postromantique, l'article propose une analyse comparative entre la musique et les images pour démontrer comment la musique du film sert de support privilégié au mythe wagnérien. L'article s'attarde enfin aux répercussions du subterfuge mis en scène par Herzog, qui consiste à faire d'un Juif un héros national à l'aube du nazisme.

\begin{abstract}
In his film Invincible, the German director, Werner Herzog presents the legend of Zishe Breibart, a Jew with superhuman strength. According to the author, the dramatic elements of the images filmed by Herzog and the original music of the film created by Hans Zimmer together serve to enhance the heroic dimension of the actor and call Wagnerian opera to mind. After comparing the composition process of the music in the film and post-Romantic writing, the article does a comparative analysis of the music and the pictures to illustrate how the music in the film provides focused support for the Wagnerian myth. Finally, the article describes in detail the repercussions of the subterfuge presented by Herzog, which turns a Jew into a national hero at the dawn of the Nazi era.
\end{abstract}

